\title{
The risk associated with spinal manipulation: an overview of reviews
}

Sabrina Mai Nielsen', Simon Tarp', Robin Christensen ${ }^{1}$ Henning Bliddal², Louise Klokker ${ }^{1}$ and Marius Henriksen ${ }^{3 *}$

\begin{abstract}
Background: Spinal manipulative therapy (SMT) is a widely used manual treatment, but many reviews exist with conflicting conclusions about the safety of SMT. We performed an overview of reviews to elucidate and quantify the risk of serious adverse events (SAEs) associated with SMT.

Methods: We searched five electronic databases from inception to December 8, 2015. We included reviews on any type of studies, patients, and SMT technique. Our primary outcome was SAEs. Quality of the included reviews was assessed using a measurement tool to assess systematic reviews (AMSTAR). Since there were insufficient data for calculating incidence rates of SAEs, we used an alternative approach; the conclusions regarding safety of SMT were extracted for each review, and the communicated opinion were judged by two reviewers independently as safe, harmful, or neutral/ unclear. Risk ratios (RRs) of a review communicating that SMT is safe and meeting the requirements for each AMSTAR item, were calculated.

Results: We identified 283 eligible reviews, but only 118 provided data for synthesis. The most frequently described adverse events (AEs) were stroke, headache, and vertebral artery dissection. Fifty-four reviews (46\%) expressed that SMT is safe, 15 (13\%) expressed that SMT is harmful, and 49 reviews (42\%) were neutral or unclear. Thirteen reviews reported incidence estimates for SAEs, roughly ranging from 1 in 20,000 to 1 in 250,000,000 manipulations. Low methodological quality was present, with a median of 4 of 11 AMSTAR items met (interquartile range, 3 to 6). Reviews meeting the requirements for each of the AMSTAR items (i.e. good internal validity) had a higher chance of expressing that SMT is safe.
\end{abstract}

Conclusions: It is currently not possible to provide an overall conclusion about the safety of SMT; however, the types of SAEs reported can indeed be significant, sustaining that some risk is present. High quality research and consistent reporting of AEs and SAEs are needed.

Systematic review registration: PROSPERO CRD42015030068.

\section{Background}

Spinal manipulative therapy (SMT) is a manual treatment where a vertebral joint is passively moved between the normal range of motion and the limits of its anatomic range, though a universally accepted definition does not seem to exist [1]. SMT often involves a highvelocity, low-amplitude thrust, a technique in which the joints are adjusted rapidly, often accompanied by popping sounds $[2,3]$.

\footnotetext{
* Correspondence: marius.Henriksen@regionh.dk

${ }^{3}$ Physiotherapy and Biomechanics Research Unit, The Parker Institute, Copenhagen University Hospital, Frederiksberg \& Bispebjerg, Frederiksberg 2000, Denmark

Full list of author information is available at the end of the article
}

The use of SMT dates back to 400 BCE, but during the centuries, SMT has switched between being accepted and abandoned by the medical profession [4]. Today, SMT is included in many guidelines for primary care, such as the management of non-specific low back pain [5], and several evidence-based guidelines exist on the practice of SMT [6-10]. SMT is widely used; it has been estimated that $12 \%$ of adults in the USA and Canada are attending chiropractors each year, with $80 \%$ of the visits involving SMT [11, 12], and use of SMT has been increasing in the past several decades [13]. Various professional groups are performing SMT including chiropractors, osteopaths and manual therapists [14]. SMT is used for a wide range of diseases and conditions with frequent indications being neck and back pain [13]. 
Patient satisfaction is high [13], but the evidence on the effectiveness of SMT from randomized controlled trials (RCTs) is often unconvincing [14-17].

As with all interventions, there are risks associated with SMT. Possible harmful outcomes of SMT includes, but are not limited to, headache, radiating discomfort and fatigue [18], which are often transient, but also more serious events such as death, stroke, paralysis and fractures [19-22]. What the patients define as mild, moderate and major AEs depend on the severity of the pain or symptom, the impact on their function, the duration and by ruling out other causes for the AEs [23]. Currently, the knowledge about the risk of harms associated with SMT is fragmented since an enormous amount of literature exists on the topic, but with different conclusions. For instance, two retrospective population-based studies have suggested an association between vertebrobasilar strokes and chiropractic care (which usually involves spinal manipulation), but also a similar association with primary care physician visits [24, 25]. Another study concluded that SMT is independently associated with vertebral artery dissection [26]. Thus, uncertainty arises when single studies are reviewed, and there is a need for an overview of the field. To our knowledge, no one has provided a complete overview of what is known about the safety of SMT. Therefore, we performed an overview of reviews to elucidate and quantify the risk of serious adverse events (SAEs) associated with SMT regardless of the indications for the treatment.

\section{Methods}

A brief protocol was registered in the International Prospective Register of Systematic Reviews (PROSPERO: CRD42015030068) prior to the initiation of this overview [see protocol in Additional file 1]. This review was reported according to PRISMA harms [27] [see the completed checklist in Additional file 2].

\section{Literature search}

We searched Cochrane Database of Systematic Reviews, Cochrane Database of Abstracts of Reviews of Effects (DARE), Cochrane Health Technology Assessment Database (HTA), MEDLINE via PubMed (from 1966) and EMBASE via Ovid (from 1974). The original search was conducted on December 8, 2015 and updated on January 10, 2017, and no date restrictions were used. Our main search terms consisted of the terms spinal adjustment, chiropractic, and spine -, spinal -, lumbar -, back -, neck -, cervical -, thrust -, or osteopath manipulation, in addition to the MeSH term 'Manipulation, Chiropractic'. Our systematic review filter included the terms Cochrane, CENTRAL,
MEDLINE, EMBASE, pubmed, search, systematic review, meta-analysis, comparative effectiveness, indirect - and mixed treatment comparison, and systematic literature [see Additional file 3, showing the search strategy used]. References from relevant reviews, overviews of reviews and relevant national clinical guidelines were checked to identify additional relevant reviews.

\section{Study selection}

We included official health technology assessment reports and peer-reviewed reviews of studies of any type (including cohorts, case reports, etc.) that examine individuals receiving SMT. We did not require the SMT to be within a certain definition but relied on the definitions used by the review authors. No restrictions were put on the age, nationality, gender or health status of the population, or length of follow-up of the study. The control could be sham, placebo, any or none. At least an abstract in English, Danish, Swedish or Norwegian had to be available. For inclusion in the synthesis, data on AEs was required.

In order to ensure that the included reviews were conducted in a systematic manner, a criterion for inclusion was to include the following two items from a measurement tool to assess systematic reviews (AMSTAR): 'were two or more electronic sources searched?' and 'was the scientific quality of the included studies assessed and documented?' $[28,29]$, as done by other overview authors [30, 31]. Since no commonly accepted quality assessment tool exists for case reports, case series, cross-sectional studies or surveys, quality assessments of these study types were not required.

One reviewer (SMN) screened titles and abstracts, and subsequently reviewed full texts to identify relevant reviews for the overview. A second reviewer $(\mathrm{MH})$ was consulted when the basis for decision making was not clear. We contacted authors of studies that could not be retrieved in full text.

\section{Data extraction}

The same reviewer $(\mathrm{SMN})$ performed the data extraction, and the same second reviewer $(\mathrm{MH})$ was consulted, when the basis for decision making was not clear. When possible, we extracted only data for patients receiving SMT, when other interventions were included in a review.

The primary outcome was SAEs defined as conditions requiring hospital admission (or mortality) [32], and the secondary outcome was any AEs reported. AEs were defined as 'any untoward occurrence that may present during treatment' [32]. If the severity of an $\mathrm{AE}$ was not defined in the review, one reviewer $(\mathrm{MH})$ rated the severity of the reported AEs, and when the basis for rating 
was unclear, another reviewer (HB) was consulted. No attempt was made to contact authors of reviews or primary studies to obtain missing data.

It was pre-specified in our protocol that the AEs and SAEs should be summarized for each review with a subsequent synthesis and meta-analysis. However, the available data on AEs and SAEs were too heterogeneously and insufficiently reported. Instead, we appraised the communicated opinions of each review concerning the safety of SMT based on their conclusions regarding the AEs and SAEs. This was done by two reviewers independently (SMN, LK), who judged the communicated opinions as either 'safe', 'neutral/unclear' or 'harmful', based on the qualitative impression the reviewers had when reading the conclusions. The reviewers had no opinion about the safety/harmfulness of SMT before commencing the judgements. Cohen's weighted Kappa was calculated for the agreement between the reviewers, with a value of $0.40-0.59$ indicating 'fair agreement', 0.60-0.74 indicating 'good agreement' and $\geq 0.75$ indicating 'excellent agreement' [33]. Disagreements were resolved by a third reviewer $(\mathrm{MH})$.

\section{Quality assessment}

One reviewer (SMN) assessed the methodological quality of each review using the AMSTAR tool [28, 29]. AMSTAR consists of 11 criteria, where each was given one of the ratings: 'yes' (clearly done), 'can't answer' (unclear if completed), 'no' (clearly not done) or 'not applicable'. A second reviewer $(\mathrm{MH})$ was consulted when the basis for decision making was not clear. We calculated a summary score by awarding each 'yes' with one point for each review [28]. A score of 0-4 is often classified as low quality, 5-8 as moderate quality and 9-11 as high [34].

We did not assess the quality of the evidence presented by each of the reviews. However, if a quality of evidence assessment (such as a GRADE assessment) was reported in the reviews, the approach and result were extracted.

\section{Data analysis}

To get an 'objective' measure of our confidence in the subjectively judged communicated opinions, we assessed whether a pattern of communicated opinions could be identified according to methodological quality of the reviews (i.e. AMSTAR). This was done by calculating a risk ratio (RR) of a review communicating the opinion 'safe' when meeting the requirements for each AMSTAR item, and a RR of the opinion of a review communicating 'harmful' when meeting the requirements for each AMSTAR item. The decision to conduct this assessment and subsequent analyses were, however, done post hoc.
Risk estimates for SAEs reported in the reviews are presented in a separate table, and a matrix was constructed showing which studies the estimates from each review were based on. All statistical analyses were performed using the statistical software R, version 3.2.3 ( $\mathrm{R}$ Foundation for Statistical Computing).

\section{Results \\ Study selection}

The reviewer screened 2305 records and identified 841 potentially eligible records (Fig. 1). Thirteen authors were contacted regarding studies that could not be retrieved in full-text. Twelve authors responded of which 9 were able to provide full-text versions. Reviewing full-texts resulted in 257 records describing 252 reviews eligible for the overview [see Additional file 4 for a list of the excluded reviews]. From reference lists, we further identified 8 records on 6 eligible reviews. In total, 265 records describing 258 reviews were included in the overview [see Additional file 5 for a list of the 258 included studies]; of these, 110 records describing 104 reviews were included in the synthesis. The updated search resulted in screening of 267 additional records, identifying 68 potentially eligible records. Of these, 26 records describing 25 reviews were eligible for the overview, and 15 records describing 14 reviews were included in the synthesis. In total, 283 reviews were included in the overview, of which 118 reviews were included in the synthesis.

\section{Characteristics of the included reviews}

The main characteristics of the 118 reviews included are presented in Table 1 [see Table, Additional file 6, which shows further study characteristics]. The included reviews consisted of 13 Cochrane reviews [14-17, 35-46], 41 other reviews including only RCTs [47-87], 53 reviews including study types other than RCTs [88-140], 3 guidelines $[9,141-143]$ and 8 health technology assessments [144-154].

The vast majority of the reviews investigated SMT (either as the only intervention or as a separate subgroup). Some of these reviews further specified SMT as cervical, thoracic or lumbar SMT (21 reviews [46, $47,49,54,57,65,91,96,103,105,114,115,119$, $121,123,125-127,134,136,150])$. Other reviews did not further specify than 'manipulation' (10 reviews [36, 66, 70-73, 79, 93, 101, 107]), 'osteopathic manipulative treatment/therapy' (8 reviews [38, 52, 56, $64,81,82,116,139])$, and 'chiropractic care/interventions' (5 reviews [67, 98-100, 137]).

The populations most frequently studied were patients with cervical pain, low back pain or headache (based on a word count after categorization by the authors; Table 2). For 81 of the reviews, the main 


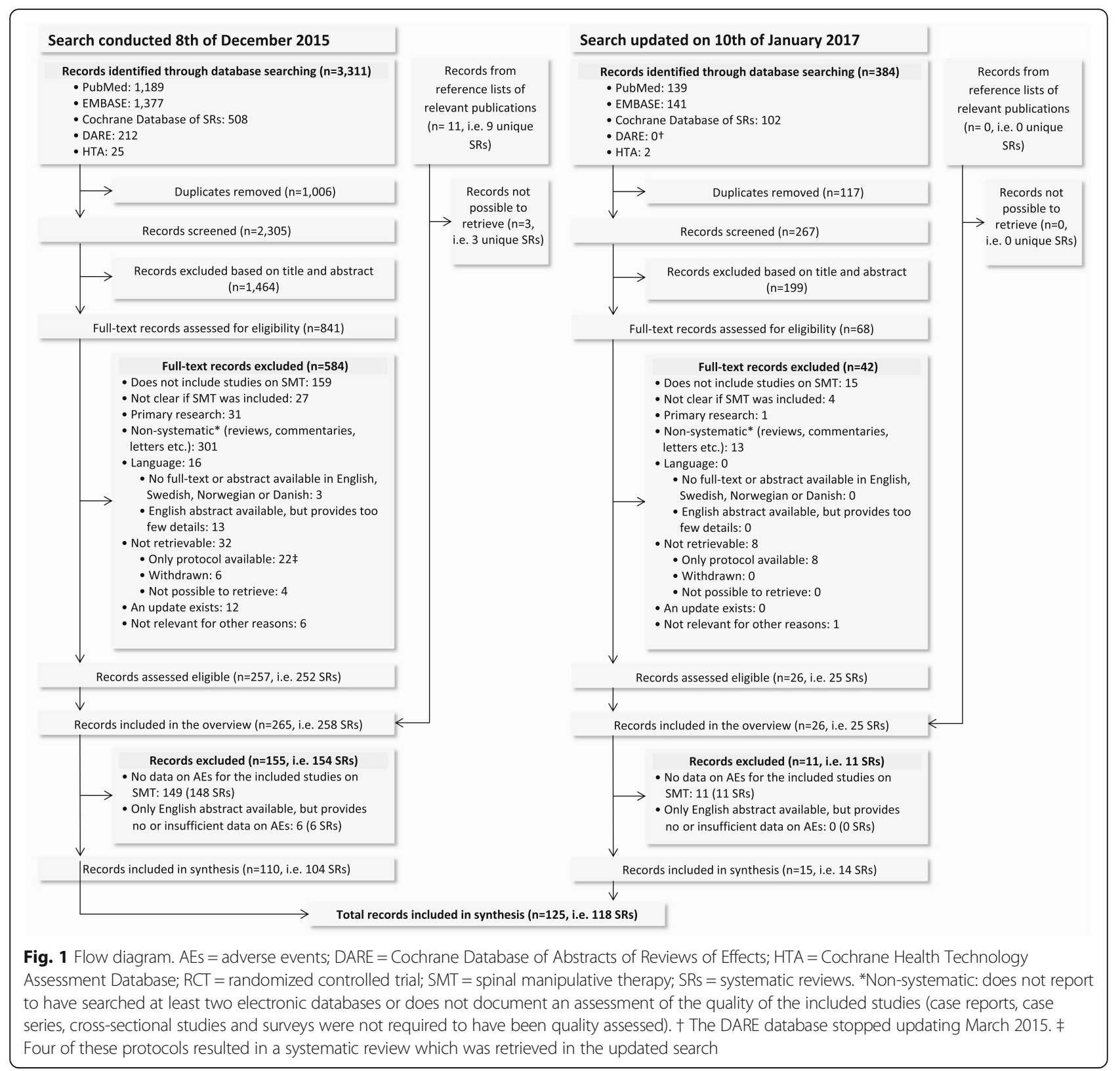

aim was to investigate efficacy (benefit), for 29 of the reviews, the main aim was to investigate AEs, and for the remaining 8 , the aim was to investigate both.

A word count of the reported AEs and SAEs showed that the most frequently used term describing AEs/SAEs in the reviews was stroke (counted after categorization by the authors; Table 3). However, it should be noted that a very common subject in the discussion sections was the poor reporting of AEs in the primary studies and the possible risk of underreporting. Thirteen of the reviews reported estimates for the incidence of SAEs, and also here, many of the reviews noted that these were rough estimates [see Table, Additional file 6, which includes conclusions extracted from each reviews].

\section{The methodological quality of included reviews}

None of the reviews met the requirements for all 11 AMSTAR items (Table 4). The median number of 'yes' was 4 (interquartile range, 3 to 6), with a minimum and maximum of 0 and 9 'yes' respectively. Only very few reviews had combined (e.g. in meta-analysis or other means of synthesis) the findings of AEs and SAEs or done this in an appropriate way; hence, item 9 was not applicable in most cases. One of the reviews made an attempt to assess the publication bias specifically for AEs 
Table 1 Summary of findings for spinal manipulative therapy

\begin{tabular}{|c|c|c|c|c|c|c|c|}
\hline Year & Authors & $\begin{array}{l}\text { Main } \\
\text { objectives }\end{array}$ & $\begin{array}{l}\text { Number of } \\
\text { pts receiving } \\
\text { intervention }\end{array}$ & $\begin{array}{l}\text { Any AEs } \\
\text { reported }\end{array}$ & $\begin{array}{l}\text { Any SAEs } \\
\text { reported }\end{array}$ & $\begin{array}{l}\text { Estimate for the incidence } \\
\text { of SAES }\end{array}$ & $\begin{array}{l}\text { Communicated } \\
\text { opinion }\end{array}$ \\
\hline 2016 & Blanchette, M. A. et al. [80] & Effect & 460 & Yes & No & No & Safe \\
\hline 2016 & Cerritelli, F. et al. [81] & Effect & 31 & No & No & No & Neutral/unclear \\
\hline 2016 & Chou, R. et al. [154] & Both & $>1100$ & Yes & No & No & Neutral/unclear \\
\hline 2016 & Church, E. W. et al. [137] & AES & 5934 & Unclear & Yes & No & Safe \\
\hline 2016 & Hall, H. et al. [83] & Effect & 249 & No & No & No & Neutral/unclear \\
\hline 2016 & Page Matthew, J. et al. [46] & Both & 117 & No & No & No & Safe \\
\hline 2016 & Ruddock, J. K. et al. [84] & Effect & 261 & Yes & No & No & Neutral/unclear \\
\hline 2016 & Ruffini, N. et al. [139] & Effect & 52 & No & No & No & Neutral/unclear \\
\hline 2016 & Varatharajan, S. et al. [85] & Effect & 129 & Yes & No & No & Safe \\
\hline 2016 & Wearing, J. et al. [140] & Effect & 55 & Yes & No & No & Safe \\
\hline 2016 & Wong, J. J. et al. [86] & Effect & 369 & Yes & No & No & Safe \\
\hline 2016 & Yao, M. et al. [87] & Effect & 1135 & Yes & Yes & No & Safe \\
\hline 2015 & Cicchintti L. et al. [116] & Effect & NA & Yes & No & No & Safe \\
\hline 2015 & Franke, H. et al. [82] & Both & 67 & Yes & No & No & Safe \\
\hline 2015 & Gross A. J. et al. [36] & Effect & NA & Yes & No & No & Safe \\
\hline 2015 & Liddle S. D. \& Pennick V. [35] & Effect & 289 & Unclear & No & No & Safe \\
\hline 2015 & Posadzki, P. et al. [138] & Effect & NA & Unclear & Unclear & No & Neutral/unclear \\
\hline 2015 & $\begin{array}{l}\text { Puentedura E. J. \& O'Grady W. } \\
\text { H. [134] }\end{array}$ & AEs & 10 & Yes & Yes & No & Harmful \\
\hline 2015 & Southerst D. et al. [49] & Effect & 98 & Yes & No & No & Safe \\
\hline 2015 & Yuan Q.-L. et al. [48] & Effect & 208 & No & No & No & Safe \\
\hline 2015 & Zhu L. et al. [47] & Effect & NA & No & No & No & Neutral/unclear \\
\hline 2014 & Bryans R. et al. [9] & Both & 513 & Yes & No & No & Safe \\
\hline 2014 & Clar C. et al. [101] & Effect & NA & Yes & Yes & No & Neutral/unclear \\
\hline 2014 & Close C. et al. [53] & Effect & NA & Yes & No & No & Neutral/unclear \\
\hline 2014 & Franke H. et al. [52] & Effect & 779 & Yes & No & No & Safe \\
\hline 2014 & Kizhakkeveettil A. et al. [51] & Effect & 1799 & Unclear & Unclear & No & Neutral/unclear \\
\hline 2014 & Page M. J. et al. [37] & Effect & 4 & No & No & No & Safe \\
\hline 2014 & Sutton D. et al. [50] & Effect & 813 & Yes & No & No & Safe \\
\hline 2014 & Todd A. J. et al. [98] & AEs & $>34,605$ & Yes & Yes & Yes & Harmful \\
\hline 2014 & Tuchin P. [130] & AES & 9 & Yes & No & No & Neutral/unclear \\
\hline 2014 & Yin P. et al. [93] & AEs & 94 & Yes & Yes & No & Harmful \\
\hline 2014 & Young J. L. et al. [105] & Effect & 539 & Yes & No & No & Safe \\
\hline 2013 & Brantingham J. W. et al. [97] & Effect & 109 & Yes & Unclear & No & Safe \\
\hline 2013 & Hebert J. J. et al. [103] & AEs & 77 & Yes & Yes & No & Neutral/unclear \\
\hline 2013 & Huisman P. A. et al. [57] & Effect & 350 & Yes & No & No & Neutral/unclear \\
\hline 2013 & Parkinson L. et al. [109] & Effect & $>520$ & No & No & No & Safe \\
\hline 2013 & Posadzki P. et al. [56] & Effect & $>448$ & Yes & No & No & Neutral/unclear \\
\hline 2013 & $\begin{array}{l}\text { Scholten-Peeters G. G. M. et al. } \\
\text { [55] }\end{array}$ & Effect & 626 & Yes & No & No & Safe \\
\hline 2013 & Schroeder J. et al. [54] & Effect & 195 & Yes & Unclear & No & Safe \\
\hline 2013 & Wynd S. et al. [126] & AEs & 901 & Unclear & Yes & No & Neutral/unclear \\
\hline 2013 & Yang M. et al. [38] & Effect & 39 & Yes & No & No & Safe \\
\hline
\end{tabular}


Table 1 Summary of findings for spinal manipulative therapy (Continued)

\begin{tabular}{|c|c|c|c|c|c|c|c|}
\hline 2012 & Brantingham J. W. et al. [102] & Effect & $>109$ & Yes & No & No & Safe \\
\hline 2012 & Dobson D. et al. [40] & Effect & 116 & No & No & No & Neutral/unclear \\
\hline 2012 & Furlan A. D. et al. $[148,149]$ & Both & NA & Yes & Yes & No & Neutral/unclear \\
\hline 2012 & Gleberzon B. J. et al. [106] & Effect & NA & Yes & Unclear & No & Safe \\
\hline 2012 & Haynes M. J. et al. [125] & AEs & NA & Unclear & Yes & No & Neutral/unclear \\
\hline 2012 & Kuczynski J. J. et al. [60] & Effect & 268 & Yes & No & No & Safe \\
\hline 2012 & Lin J. H. et al. [59] & Effect & 283 & No & No & No & Neutral/unclear \\
\hline 2012 & Posadzki P. \& Ernst E. [58] & Effect & NA & Yes & No & No & Neutral/unclear \\
\hline 2012 & Puentedura E. J. et al. [123] & AEs & 134 & Yes & Yes & No & Harmful \\
\hline 2012 & Rubinstein S. M. et al. $[15,39]$ & Effect & 1195 & Yes & No & No & Safe \\
\hline 2012 & Stuber K. A. et al. [132] & AEs & NA & Yes & Yes & No & Neutral/unclear \\
\hline 2011 & Brantingham J. et al. [104] & Effect & $>266$ & No & No & No & Neutral/unclear \\
\hline 2011 & Cross K. et al. [65] & Effect & 187 & Yes & No & No & Safe \\
\hline 2011 & Huang T. et al. [43] & Effect & 131 & Yes & No & No & Safe \\
\hline 2011 & Lystad R. P. et al. [112] & Effect & NA & Yes & No & No & Safe \\
\hline 2011 & Posadzki P. \& Ernst E. [63] & Effect & NA & Yes & No & No & Harmful \\
\hline 2011 & Posadzki P. \& Ernst E. [62] & Effect & NA & Yes & No & No & Harmful \\
\hline 2011 & Posadzki P. \& Ernst E. [61] & Effect & NA & Unclear & Unclear & No & Neutral/unclear \\
\hline 2011 & Posadzki P. \& Ernst E. [64] & Effect & NA & Yes & No & No & Neutral/unclear \\
\hline 2011 & Rubinstein S. M. et al. $[14,42]$ & Effect & 2435 & Yes & No & No & Safe \\
\hline 2011 & Walker B. F. et al. $[41,44]$ & Effect & NA & Yes & No & No & Neutral/unclear \\
\hline 2010 & Carlesso L. C. et al. [96] & AEs & NA & Yes & No & No & Neutral/unclear \\
\hline 2010 & Carnes D. et al. [88] & AES & 25,179 & Yes & Yes & Yes & Safe \\
\hline 2010 & Ernst E. [128] & AEs & 26 & Unclear & Yes & No & Harmful \\
\hline 2010 & Hahne A. J. et al. [66] & Effect & NA & No & No & No & Safe \\
\hline 2010 & Kaminskyj A. et al. [108] & Effect & NA & Yes & No & No & Neutral/unclear \\
\hline 2010 & Shin B.-C. et al. [95] & AEs & 18 & Yes & Yes & No & Harmful \\
\hline 2009 & Boudreau R. et al. [147] & Effect & $>52$ & Yes & Yes & No & Safe \\
\hline 2009 & Boudreau R. \& Spry C. [151] & Effect & 1 & No & No & No & Safe \\
\hline 2009 & Brurberg K. G. et al. [145] & Effect & $>695$ & Yes & No & No & Safe \\
\hline 2009 & Gouveia L. O. et al. [99] & AEs & $>2838$ & Yes & Yes & Yes & Harmful \\
\hline 2009 & Hunt K. J. et al. [67] & Effect & NA & Yes & No & No & Safe \\
\hline 2009 & Khorsan B. et al. [94] & Effect & $>297$ & Unclear & Yes & No & Neutral/unclear \\
\hline 2009 & Reiman M. P. et al. [110] & Effect & $>76$ & Yes & Unclear & No & Neutral/unclear \\
\hline 2008 & Miley M. L. et al. [127] & AEs & NA & Unclear & Yes & Yes & Harmful \\
\hline 2008 & Stuber K. J. \& Smith D. L. [107] & Effect & 285 & No & No & No & Neutral/unclear \\
\hline 2008 & $\begin{array}{l}\text { Vernon H. \& Humphreys B. K. } \\
\text { [68] }\end{array}$ & Effect & 178 & Yes & No & No & Safe \\
\hline 2007 & $\begin{array}{l}\text { Chou R. \& Huffman L. H. [141, } \\
\text { 143] }\end{array}$ & Effect & NA & Yes & Yes & Yes & Safe \\
\hline 2007 & Ernst E. [118] & AEs & $>924$ & Yes & Yes & No & Harmful \\
\hline 2007 & Gross A. R. et al. [71] & Effect & NA & Unclear & No & No & Safe \\
\hline 2007 & Hawk C. et al. [100] & Effect & NA & Yes & No & No & Safe \\
\hline 2007 & Luijsterburg P. A. J. et al. [70] & Effect & 175 & No & No & No & Neutral/unclear \\
\hline 2007 & Vernon H. \& Humphreys B. K. & Effect & 701 & Yes & No & No & Neutral/unclear \\
\hline
\end{tabular}


Table 1 Summary of findings for spinal manipulative therapy (Continued)

\begin{tabular}{|c|c|c|c|c|c|c|c|}
\hline 2007 & Vernon H. et al. [113] & Effect & 593 & Yes & No & No & Safe \\
\hline 2006 & Gemmell H. \& Miller P. [111] & Effect & $>79$ & Unclear & Unclear & No & Safe \\
\hline 2006 & Proctor M. et al. [16] & Effect & $>162$ & Yes & Unclear & No & Safe \\
\hline 2006 & Snelling N. J. [135] & Both & $>214$ & Yes & Yes & Yes & Neutral/unclear \\
\hline 2005 & Brown A. et al. [146] & Effect & NA & Yes & Yes & No & Safe \\
\hline 2005 & Ernst E. [133] & AEs & 14 & Yes & Yes & No & Harmful \\
\hline 2005 & Hondras M. A. et al. [17] & Effect & NA & No & No & No & Safe \\
\hline 2005 & Lisi A. J. et al. [120] & Effect & 183 & Yes & No & No & Neutral/unclear \\
\hline 2005 & Rubinstein S. M. et al. [91] & AEs & 7 & Unclear & Yes & No & Neutral/unclear \\
\hline 2004 & Brønfort G. et al. [45] & Effect & 85 & Yes & No & No & Safe \\
\hline 2004 & Ernst E. [89] & AEs & 340 & Yes & Yes & No & Neutral/unclear \\
\hline 2004 & Lenssinck M.-L. B. et al. [72] & Effect & NA & Yes & No & No & Safe \\
\hline 2004 & Oduneye F. [152] & Effect & 128 & Yes & No & No & Neutral/unclear \\
\hline 2004 & Oliphant D. [115] & AEs & NA & Yes & Yes & Yes & Safe \\
\hline 2003 & Ernst E. [90] & AEs & 2 & Yes & Yes & No & Harmful \\
\hline 2002 & Ernst E. [119] & AEs & $>4$ & Yes & Yes & No & Neutral/unclear \\
\hline 2002 & Ernst E. [136] & AEs & 42 & Yes & Yes & No & Harmful \\
\hline 2002 & Gerritsen A. A. M. et al. [74] & Effect & 45 & Yes & No & No & Safe \\
\hline 2002 & Gross A. R. et al. [142] & Both & NA & Yes & Yes & Yes & Neutral/unclear \\
\hline 2002 & Gross A. R. et al. [73] & Effect & NA & Yes & No & No & Neutral/unclear \\
\hline 2002 & Stevinson C. \& Ernst E. [124] & AEs & $>2357$ & Yes & Yes & Yes & Neutral/unclear \\
\hline 2001 & Bronfort G. et al. [76] & Effect & 400 & Yes & No & No & Safe \\
\hline 2001 & Ernst E. [129] & AEs & $>2016$ & Yes & No & No & Neutral/unclear \\
\hline 2001 & Ernst E. \& Harkness E. [75] & Effect & NA & Yes & No & No & Neutral/unclear \\
\hline 2000 & Ernst E. [77] & Effect & NA & Yes & No & No & Neutral/unclear \\
\hline 2000 & Magee D. J. et al. [117] & Effect & 10 & No & No & No & Safe \\
\hline 1999 & Fabio R. P. D. [121] & Both & 177 & Yes & Yes & No & Harmful \\
\hline 1999 & Haldeman S. M. et al. [122] & AEs & 115 & Unclear & Yes & No & Safe \\
\hline 1999 & Vernon H. et al. [78] & Effect & 176 & Yes & No & No & Neutral/unclear \\
\hline 1996 & Aker P. D. et al. [79] & Effect & NA & Yes & No & No & Neutral/unclear \\
\hline 1996 & Assendelft W. J. J. et al. [131] & AEs & $>1795$ & Yes & Yes & Yes & Neutral/unclear \\
\hline 1996 & Hurwitz E. L. et al. [144, 150] & Effect & $>935$ & Yes & Yes & Yes & Neutral/unclear \\
\hline 1995 & Dabbs V. \& Lauretti W. J. [114] & AEs & NA & Unclear & Yes & Yes & Safe \\
\hline 1992 & Shekelle P. G. et al. [92] & Effect & $>1500$ & Unclear & Yes & Yes & Neutral/unclear \\
\hline
\end{tabular}

When 'Number of patients in total' has ' $>$ ' in front, the actual number of patients is higher since incomplete data were provided by the review AEs adverse events, NA no data available, pts patients, SAEs serious adverse events

and/or SAEs; hence, this one review met the requirements for item 10 .

Furthermore, very few reviews rated the quality of the evidence for AEs and/or SAEs, with GRADE being the most frequently used tool.

\section{Serious adverse events}

The estimates for the incidence of SAEs (Table 5) were heterogeneous, as they had different units (e.g. per number of manipulations, per visits or no unit), were based on different patient types, and were obtained from different types of studies [see Table, Additional file 7, showing which studies the estimates for the incidence of SAEs are based on].

When not distinguishing between the different types of SMT treatments and assuming that one treatment or visit equals one manipulation, and leaving out the minority of estimates not specifying the units or using per patient as the unit, the estimates for the incidence of 
Table 2 The patient populations most frequently studied in the included reviews (listed after frequency shown in brackets)

1. Cervical pain (25)
2. Low back pain (18)
3. Headache (16)
4. Children/adolescents (6)
5. Asthma (4)
6. Cervical radiculopathy (4)
7. Musculoskeletal (various) (4)
8. Pregnant (4)
9. Dysmenorrhea (3)
10. Lumbar radiculopathy (3)
11. Pelvic pain (3)
12. Carpal tunnel syndrome (2)
13.Phobia (2)
14. Cervical trauma (1)
15. Chronic inflammatory disease (1)
16. Chronic obstructive pulmonary disease (1)
17. Colic (1)
18. Diversity of complaints (1)
19. Dizziness (1)
20. Frozen shoulder (1)

Table 3 The terms describing the adverse events and serious adverse events most frequently used in the reviews (listed after frequency shown in brackets)
1. Stroke (36)
2. Headache (34)
3. Vertebral artery dissection (29)
4. Increased pain (22)
5. Fatigue (18)
6. Aggravation of symptoms (17)
7. Death (17)
8. Radiculopathy (17)
9. Soreness (16)
10. Spinal cord injury (16)
11. Cauda equine syndrome (15)
12. Disc herniation (13)
13. Vertebral fracture (12)
14. Discomfort (11)
15. Minor side effects (10)
16. Stiffness (10)
17. Dizziness (9)
18. Nausea (9)
19. Vertebral dislocation (8)
20. Neck-stiffness (7)

SAEs ranges from 1 in 20,000 manipulations to 1 in 250,000,000 manipulations (Table 6).

Based on the conclusions of the reviews regarding AEs and SAEs, 54 reviews (46\%) expressed that SMT is safe, $15(13 \%)$ expressed that SMT is harmful and 49 reviews $(42 \%)$ were neutral or unclear regarding the safety of SMT, with a fair agreement between the two reviewers (Cohens Weighted Kappa, 0.50).

The calculations of RRs show a higher chance of a review communicating that SMT is safe, when having a higher methodological quality, compared to reviews of lower methodological quality (statistically significant for the AMSTAR items 5, 7 and 8; Table 7). And vice versa, there is a lower chance of a review communicating that SMT is harmful, when it has a lower methodological quality.

\section{Reviews specifically investigating adverse events}

When only considering the subset of reviews, where the objective was to investigate AEs (37 reviews), then 8 reviews (22\%) expressed that SMT is safe, 13 reviews (35\%) expressed that SMT is harmful and 16 reviews (43\%) were neutral or unclear regarding the safety of SMT. Hence, there is a tendency that a bigger proportion of these reviews are expressing that SMT is harmful compared to the full sample of reviews. The calculations of RRs did not obtain enough power to show any statistically significant RRs [see Table, Additional file 8, which shows the calculations of RRs]. The possibility of a causal relationship between SMT and SAEs was specifically investigated in six of the included reviews [89, 90, $118,124,127,133$ ] (Table 8). Five of these had for each case report or case series assessed the likelihood of causality [89, 90, 118, 124, 133]. In all cases, 'certain' was not the single most used rating. Miley et al. [127] used another approach and concluded weak to moderate strength of evidence for a causal relationship between cervical SMT and vertebral artery dissection, and expressed that comprehensive prospective studies are needed to further examine this relationship.

\section{Discussion}

In this overview, the included reviews did not provide sufficient data for synthesis, and therefore it is currently not possible to provide an overall estimate for the risk of SAEs associated with SMT. Of the few reviews providing estimates for the incidence of SAEs, no reliable single estimate was provided, and it was not possible to identify any agreement regarding the safety of SMT across the included reviews. Interestingly, we found indications that reviews with higher methodological quality generally used language suggesting SMT to be safer (or less harmful). However, when analysing this across the reviews whose objective was to investigate safety, this could not 
Table 4 Methodological quality of included reviews assessed with AMSTAR

\begin{tabular}{|c|c|c|c|c|c|c|c|c|c|c|c|c|c|}
\hline Year & Authors & 1 & 2 & 3 & 4 & 5 & 6 & 7 & 8 & 9 & 10 & 11 & Total score ${ }^{a}$ \\
\hline 2016 & Blanchette, M. A. et al. [80] & Yes & Yes & Yes & Yes & No & Yes & Yes & Yes & NA & No & No & 7 \\
\hline 2016 & Cerritelli, F. et al. [81] & No & Yes & Yes & Yes & No & Yes & Yes & Yes & NA & No & No & 6 \\
\hline 2016 & Chou, R. et al. [153] & Yes & Yes & Yes & Yes & Yes & Yes & Yes & No & NA & No & Yes & 8 \\
\hline 2016 & Church, E. W. et al. [137] & No & No & Yes & No & No & No & Yes & Yes & Yes & Yes & No & 5 \\
\hline 2016 & Hall, H. et al. [83] & Yes & Yes & Yes & No & Yes & Yes & Yes & Yes & NA & No & No & 7 \\
\hline 2016 & Page Matthew, J. et al. [46] & Yes & Yes & Yes & Yes & Yes & Yes & Yes & Yes & NA & No & Yes & 9 \\
\hline 2016 & Ruddock, J. K. et al. [84] & Yes & Yes & Yes & No & Yes & Yes & Yes & Yes & NA & No & No & 7 \\
\hline 2016 & Ruffini, N. et al. [139] & No & Yes & Yes & Yes & Yes & Yes & Yes & Yes & NA & No & No & 7 \\
\hline 2016 & Varatharajan, S. et al. [85] & Yes & No & No & No & No & Yes & Yes & No & NA & No & No & 3 \\
\hline 2016 & Wearing, J. et al. [140] & No & Yes & Yes & No & No & Yes & Yes & Yes & NA & No & No & 5 \\
\hline 2016 & Wong, J. J. et al. [86] & Yes & No & No & No & No & Yes & Yes & No & NA & No & No & 3 \\
\hline 2016 & Yao, M. et al. [87] & Yes & Yes & Yes & Yes & No & Yes & Yes & Yes & NA & No & No & 7 \\
\hline 2015 & Cicchintti L. et al. [116] & No & Yes & Yes & Yes & No & Yes & Yes & Yes & NA & No & Yes & 7 \\
\hline 2015 & Franke, H. et al. [82] & No & Unclear & Yes & Yes & Yes & Yes & Yes & Yes & NA & No & No & 6 \\
\hline 2015 & Gross A. J. et al. [36] & Yes & Yes & Yes & Yes & Yes & Yes & No & No & Yes & No & No & 7 \\
\hline 2015 & Liddle S. D. \& Pennick V. [35] & No & Yes & Yes & Yes & Yes & Yes & Yes & Yes & NA & No & Yes & 8 \\
\hline 2015 & Posadzki, P. et al. [138] & Yes & No & Yes & No & No & Yes & Yes & No & NA & No & No & 4 \\
\hline 2015 & Puentedura E. J. \& O'Grady W. H. [134] & No & No & Yes & No & No & Yes & No & No & No & NA & No & 2 \\
\hline 2015 & Southerst D. et al. [49] & Yes & Yes & Yes & Yes & No & Yes & Yes & Yes & NA & No & No & 7 \\
\hline 2015 & Yuan Q.-L. et al. [48] & No & Unclear & Yes & Yes & No & Yes & Yes & Yes & NA & No & No & 5 \\
\hline 2015 & Zhu L. et al. [47] & No & Yes & Yes & Yes & No & Yes & Yes & Yes & NA & No & No & 6 \\
\hline 2014 & Bryans R. et al. [9] & No & No & Yes & No & No & Yes & Yes & No & Yes & No & No & 4 \\
\hline 2014 & Clar C. et al. [101] & No & Yes & Yes & No & No & No & Yes & Yes & NA & No & No & 4 \\
\hline 2014 & Close C. et al. [53] & Yes & No & Yes & Yes & No & Yes & Yes & Yes & NA & No & No & 6 \\
\hline 2014 & Franke H. et al. [52] & No & Yes & Yes & Yes & Yes & Yes & Yes & Yes & NA & No & No & 7 \\
\hline 2014 & Kizhakkeveettil A. et al. [51] & No & Yes & Yes & No & No & Yes & Yes & No & NA & No & No & 4 \\
\hline 2014 & Page M. J. et al. [37] & Yes & Yes & Yes & Yes & Yes & Yes & Yes & Yes & NA & No & Yes & 9 \\
\hline 2014 & Sutton D. et al. [50] & Yes & Yes & Yes & No & No & Yes & Yes & Yes & NA & No & No & 6 \\
\hline 2014 & Todd A. J. et al. [98] & No & No & Yes & Yes & No & Yes & No & No & NA & No & No & 3 \\
\hline 2014 & Tuchin P. [130] & No & No & Yes & No & No & Yes & No & No & NA & NA & No & 2 \\
\hline 2014 & Yin P. et al. [93] & No & Yes & No & No & No & Yes & No & No & NA & NA & No & 2 \\
\hline 2014 & Young J. L. et al. [105] & No & No & No & No & No & Yes & Yes & Yes & NA & No & No & 3 \\
\hline 2013 & Brantingham J. W. et al. [97] & No & No & Yes & Yes & No & Yes & Yes & Yes & NA & No & No & 5 \\
\hline 2013 & Hebert J. J. et al. [103] & No & No & Yes & No & No & Yes & No & No & NA & NA & No & 2 \\
\hline 2013 & Huisman P. A. et al. [57] & No & No & Yes & No & Yes & Yes & Yes & Yes & NA & No & No & 5 \\
\hline 2013 & Parkinson L. et al. [109] & No & Yes & No & No & No & Yes & Yes & Yes & NA & No & No & 4 \\
\hline 2013 & Posadzki P. et al. [56] & Yes & Yes & Yes & No & No & Yes & Yes & Yes & NA & No & No & 6 \\
\hline 2013 & Scholten-Peeters G. G. M. et al. [55] & No & Yes & Yes & No & No & Yes & Yes & Yes & NA & No & No & 5 \\
\hline 2013 & Schroeder J. et al. [54] & No & No & No & No & Yes & Yes & Yes & Yes & NA & No & No & 4 \\
\hline 2013 & Wynd S. et al. [126] & No & Yes & No & No & Yes & No & No & No & No & No & No & 2 \\
\hline 2013 & Yang M. et al. [38] & Yes & Yes & Yes & No & Yes & Yes & Yes & Yes & NA & No & Yes & 8 \\
\hline 2012 & Brantingham J. W. et al. [102] & No & No & No & No & No & Yes & Yes & Yes & NA & No & No & 3 \\
\hline 2012 & Dobson D. et al. [40] & Yes & Yes & Yes & Yes & Yes & Yes & Yes & Yes & NA & No & Yes & 9 \\
\hline
\end{tabular}


Table 4 Methodological quality of included reviews assessed with AMSTAR (Continued)

\begin{tabular}{|c|c|c|c|c|c|c|c|c|c|c|c|c|c|}
\hline 2012 & Furlan A. D. et al. $[148,149]$ & No & Yes & Yes & No & No & Yes & Yes & Yes & NA & No & No & 5 \\
\hline 2012 & Gleberzon B. J. et al. [106] & No & No & Yes & No & No & Yes & Yes & Yes & NA & No & No & 4 \\
\hline 2012 & Haynes M. J. et al. [125] & Yes & No & Yes & No & No & No & Yes & Yes & NA & NA & No & 4 \\
\hline 2012 & Kuczynski J. J. et al. [60] & No & Yes & No & No & No & Yes & Yes & No & NA & No & No & 3 \\
\hline 2012 & Lin J. H. et al. [59] & No & Yes & Yes & No & No & Yes & Yes & Yes & NA & No & No & 5 \\
\hline 2012 & Posadzki P. \& Ernst E. [58] & Yes & No & Yes & No & No & Yes & Yes & Yes & NA & No & No & 5 \\
\hline 2012 & Puentedura E. J. et al. [123] & No & Yes & Yes & No & No & Yes & No & No & Yes & NA & No & 4 \\
\hline 2012 & Rubinstein S. M. et al. $[15,39]$ & Yes & No & Yes & Yes & Yes & Yes & Yes & Yes & Yes & No & Yes & 9 \\
\hline 2012 & Stuber K. A. et al. [132] & No & No & Yes & No & Yes & No & Yes & No & NA & NA & No & 3 \\
\hline 2011 & Brantingham J. et al. [104] & No & No & No & No & No & Yes & Yes & Yes & NA & No & No & 3 \\
\hline 2011 & Cross K. et al. [65] & No & Yes & Yes & No & Yes & Yes & Yes & Yes & NA & No & No & 6 \\
\hline 2011 & Huang T. et al. [43] & Yes & Yes & Yes & No & Yes & Yes & Yes & Yes & NA & No & No & 7 \\
\hline 2011 & Lystad R. P. et al. [112] & No & No & Yes & No & Yes & Yes & Yes & Yes & NA & No & No & 5 \\
\hline 2011 & Posadzki P. \& Ernst E. [63] & No & No & Yes & No & No & Yes & Yes & Yes & NA & No & No & 4 \\
\hline 2011 & Posadzki P. \& Ernst E. [62] & No & No & Yes & No & No & Yes & Yes & Yes & NA & No & No & 4 \\
\hline 2011 & Posadzki P. \& Ernst E. [61] & No & No & Yes & No & No & Yes & Yes & Yes & NA & No & Yes & 5 \\
\hline 2011 & Posadzki P. \& Ernst E. [64] & No & Yes & Yes & Yes & No & Yes & Yes & Yes & NA & No & No & 6 \\
\hline 2011 & Rubinstein S. M. et al. [14, 42] & Yes & Yes & Yes & Yes & Yes & Yes & Yes & Yes & NA & No & Yes & 9 \\
\hline 2011 & Walker B. F. et al. [41, 44] & Yes & Yes & Yes & Yes & Yes & Yes & Yes & Yes & NA & No & No & 8 \\
\hline 2010 & Carlesso L. C. et al. [96] & No & Yes & No & Yes & No & Yes & Yes & Yes & Yes & No & No & 6 \\
\hline 2010 & Carnes D. et al. [88] & No & No & Yes & No & No & No & Yes & No & No & No & No & 2 \\
\hline 2010 & Ernst E. [128] & No & No & Yes & No & No & Yes & No & No & NA & NA & No & 2 \\
\hline 2010 & Hahne A. J. et al. [66] & No & Yes & Yes & No & No & Yes & Yes & Yes & NA & No & No & 5 \\
\hline 2010 & Kaminskyj A. et al. [108] & No & No & Yes & Yes & Yes & Yes & Yes & Yes & NA & No & No & 6 \\
\hline 2010 & Shin B.-C. et al. [95] & No & Unclear & Yes & Yes & No & Yes & No & No & NA & NA & No & 3 \\
\hline 2009 & Boudreau R. et al. [147] & No & No & No & No & No & Yes & No & No & NA & No & No & 1 \\
\hline 2009 & Boudreau R. \& Spry C. [151] & No & Unclear & No & No & Yes & Yes & No & No & NA & No & No & 2 \\
\hline 2009 & Brurberg K. G. et al. [145] & No & No & Yes & No & Yes & No & Yes & Yes & NA & No & No & 4 \\
\hline 2009 & Gouveia L. O. et al. [99] & No & No & Yes & No & No & Yes & No & No & No & NA & No & 2 \\
\hline 2009 & Hunt K. J. et al. [67] & No & Yes & Yes & No & No & Yes & Yes & Yes & NA & No & No & 5 \\
\hline 2009 & Khorsan B. et al. [94] & No & No & Yes & No & No & Yes & Yes & Yes & NA & No & No & 4 \\
\hline 2009 & Reiman M. P. et al. [110] & No & No & Yes & Yes & No & Yes & Yes & Yes & NA & No & No & 5 \\
\hline 2008 & Miley M. L. et al. [127] & No & No & No & No & No & No & No & No & No & No & No & 0 \\
\hline 2008 & Stuber K. J. \& Smith D. L. [107] & No & No & Yes & Yes & Yes & Yes & Yes & Yes & NA & No & No & 6 \\
\hline 2008 & Vernon H. \& Humphreys B. K. [68] & No & No & Yes & No & No & Yes & Yes & Yes & NA & No & No & 4 \\
\hline 2007 & Chou R. \& Huffman L. H. [141, 143] & No & Unclear & Yes & No & Yes & Yes & Yes & Yes & No & No & No & 5 \\
\hline 2007 & Ernst E. [118] & No & No & Yes & No & No & Yes & No & No & NA & NA & No & 2 \\
\hline 2007 & Gross A. R. et al. [71] & No & No & No & Yes & No & No & Yes & No & NA & No & No & 2 \\
\hline 2007 & Hawk C. et al. [100] & No & No & Yes & No & No & Yes & Yes & Yes & NA & No & No & 4 \\
\hline 2007 & Luijsterburg P. A. J. et al. [70] & No & No & No & No & No & Yes & Yes & Yes & NA & No & No & 3 \\
\hline 2007 & Vernon H. \& Humphreys B. K. [69] & No & Unclear & No & No & No & Yes & Yes & Yes & NA & No & No & 3 \\
\hline 2007 & Vernon H. et al. [113] & No & No & Yes & No & Yes & Yes & Yes & Yes & NA & No & No & 5 \\
\hline 2006 & Gemmell H. \& Miller P. [111] & No & No & Yes & No & No & Yes & Yes & Yes & NA & No & No & 4 \\
\hline 2006 & Proctor M. et al. [16] & Yes & Yes & Yes & Yes & Yes & Yes & Yes & Yes & NA & No & No & 8 \\
\hline
\end{tabular}


Table 4 Methodological quality of included reviews assessed with AMSTAR (Continued)

\begin{tabular}{|c|c|c|c|c|c|c|c|c|c|c|c|c|c|}
\hline 2006 & Snelling N. J. [135] & No & No & Yes & No & No & Yes & Yes & Yes & NA & No & No & 4 \\
\hline 2005 & Brown A. et al. [146] & No & Yes & Yes & No & Yes & Yes & Yes & Yes & NA & No & No & 6 \\
\hline 2005 & Ernst E. [133] & No & No & Yes & No & No & Yes & No & No & NA & NA & No & 2 \\
\hline 2005 & Hondras M. A. et al. [17] & No & Yes & Yes & Yes & Yes & Yes & Yes & Yes & NA & No & No & 7 \\
\hline 2005 & Lisi A. J. et al. [120] & No & No & Yes & No & No & Yes & Yes & Yes & NA & No & No & 4 \\
\hline 2005 & Rubinstein S. M. et al. [91] & No & Yes & Yes & No & No & Yes & No & No & NA & No & No & 3 \\
\hline 2004 & Brønfort G. et al. [45] & Yes & Yes & Yes & No & Yes & Yes & Yes & Yes & NA & No & No & 7 \\
\hline 2004 & Ernst E. [89] & No & No & Yes & No & No & Yes & No & No & NA & No & No & 2 \\
\hline 2004 & Lenssinck M.-L. B. et al. [72] & No & Yes & Yes & No & Yes & Yes & Yes & Yes & NA & No & No & 6 \\
\hline 2004 & Oduneye F. [152] & No & No & No & No & No & Yes & No & No & NA & No & No & 1 \\
\hline 2004 & Oliphant D. [115] & No & No & Yes & No & No & Yes & Yes & No & No & No & No & 3 \\
\hline 2003 & Ernst E. [90] & No & No & Yes & No & No & Yes & No & No & NA & No & No & 2 \\
\hline 2002 & Ernst E. [119] & No & No & Yes & No & No & No & No & No & NA & No & No & 1 \\
\hline 2002 & Ernst E. [136] & No & No & Yes & No & No & Yes & No & No & NA & NA & No & 2 \\
\hline 2002 & Gerritsen A. A. M. et al. [74] & No & Yes & Yes & No & No & Yes & Yes & Yes & NA & No & No & 5 \\
\hline 2002 & Gross A. R. et al. [142] & No & Yes & Yes & No & No & Yes & Yes & Yes & No & No & No & 5 \\
\hline 2002 & Gross A. R. et al. [73] & No & Yes & No & No & No & Yes & Yes & Yes & NA & No & No & 4 \\
\hline 2002 & Stevinson C. \& Ernst E. [124] & No & No & Yes & No & No & Yes & No & No & No & No & No & 2 \\
\hline 2001 & Bronfort G. et al. [76] & No & No & No & No & Yes & Yes & Yes & Yes & NA & No & No & 4 \\
\hline 2001 & Ernst E. [129] & No & No & No & No & No & Yes & No & No & NA & No & No & 1 \\
\hline 2001 & Ernst E. \& Harkness E. [75] & No & No & Yes & No & No & Yes & Yes & Yes & NA & No & No & 4 \\
\hline 2000 & Ernst E. [77] & No & No & No & No & No & Yes & No & No & NA & No & No & 1 \\
\hline 2000 & Magee D. J. et al. [117] & No & Unclear & Yes & No & No & Yes & Yes & Yes & NA & No & No & 4 \\
\hline 1999 & Fabio R. P. D. [121] & No & No & Yes & No & Yes & Yes & No & No & No & No & No & 3 \\
\hline 1999 & Haldeman S. M. et al. [122] & No & No & Yes & No & No & Yes & No & No & NA & No & No & 2 \\
\hline 1999 & Vernon H. et al. [78] & No & No & Yes & No & No & Yes & Yes & Yes & NA & No & No & 4 \\
\hline 1996 & Aker P. D. et al. [79] & No & Unclear & Unclear & Yes & No & No & Yes & Yes & NA & No & No & 3 \\
\hline 1996 & Assendelft W. J. J. et al. [131] & No & No & Unclear & Unclear & No & No & No & No & No & No & No & 0 \\
\hline 1996 & Hurwitz E. L. et al. [144, 150] & No & No & No & No & No & No & Yes & Yes & No & No & No & 2 \\
\hline 1995 & Dabbs V. \& Lauretti W. J. [114] & No & No & No & No & No & Yes & No & No & No & No & No & 1 \\
\hline \multirow[t]{2}{*}{1992} & Shekelle P. G. et al. [92] & No & No & Yes & Yes & No & Yes & Yes & Yes & NA & No & No & 5 \\
\hline & Total number of 'yes' for each item & 25 & 47 & 92 & 33 & 35 & 105 & 89 & 78 & 6 & 1 & 10 & \\
\hline
\end{tabular}

AMSTAR A Measurement Tool to Assess Systematic Reviews, NA not applicable

'The total score is the number of 'yes' for each review. It was calculated giving one point for each 'yes' given for the 11 items. 1 . Was an 'a priori' design provided? 2. Was there duplicate study selection and data extraction? 3. Was a comprehensive literature search performed? 4 . Was the status of publication (i.e. grey literature) used as an inclusion criterion? 5. Was a list of studies (included and excluded) provided? 6. Were the characteristics of the included studies provided? 7. Was the scientific quality of the included studies assessed and documented? 8 . Was the scientific quality of the included studies used appropriately in formulating conclusions? 9. Were the methods used to combine the findings of studies for AEs appropriate? 10. Was the likelihood of publication bias assessed for AEs? 11. Was the conflict of interest included?

be replicated. In the few reviews assessing the likelihood of a causal relationship between SMT and SAEs, this relationship was not in all cases certain. However, it should be noted that these assessments were based on case reports and case series, which cannot determine causality.

This overview is to our knowledge, the most comprehensive overview conducted on SMT, by including more than 100 reviews on SMT, and the only one with a sole focus on the safety aspects of SMT. Our intention was to provide an overview of all SAEs from SMT regardless of the indications for the treatment, but our overview especially covers patients with cervical pain, low back pain and headache, which were the most frequently studied populations. The most frequently mentioned AEs/SAEs across the 118 reviews ranged from minor events, such as soreness, to significant events, such as spinal cord injury and death. While some of these events may to a 
Table 5 Estimates for the incidence of serious adverse events following spinal manipulative therapy

\begin{tabular}{lll}
\hline Year Author & Estimates \\
\hline 2014 Todd A. J. et al. [98] & From a SR: 1 SAE in 250 million pediatric visits. \\
& From a discussion paper: 0 SAEs reported in $>30,000$ treatments by medical manipulators. \\
& From a pCohort: 14 cases of 'unbearably severe side effects' in 4712 treatments (0.13\%). Upper risk rate for 'serious \\
& adverse events' of approximately $0.01 \%(3 / 28,109$ consultations). \\
& Their estimation from all pCohorts: Upper $95 \%$ Cl incidence risk rate of major adverse events of 0.007\% (0/42,451) \\
& after treatment or 0.01\% (0/22,833) per patient. \\
& From RCTs: No 'major adverse events' in the 31 RCTs (which included 2281 participants who received manual \\
& therapy and 2779 who received other therapies). Upper incidence rate of major adverse events of $\sim 0.13 \%(0 / 2301)$ \\
& after manual therapy treatment.
\end{tabular}

2009 Gouveia L. O. et al. [99]

Their own synthesis (based on surveys): Between 5 strokes in 100,000 manipulations to 1.46 SAEs in 10,000,000 manipulations and 2.68 deaths in 10,000,000 manipulations.

2008 Miley M. L. et al. [127]

From a CC (which they consider the best available estimate): Approximately 1.3 cases of VAD or occlusion attributable to CMT would be observed within 1 week of manipulative therapy for every 100,000 persons $<45$ years of age receiving CMT.

From reviews: Published estimates of the incidence of VAD and stroke after range from 1 in 5.8 million to 1 in 5000.

2007 Chou R. \& Huffman L. H. $\quad$ From SRs: <1 SAE per 1 million patient visits. $[141,143]$

2006 Snelling N. J. [135]

2004 Oliphant D. [115]

2002 Gross A. R. et al. [142]

2002 Stevinson C. \& Ernst E. [124]

1996 Assendelft W. J. J. et al. [131]

1996 Hurwitz E. L. et al. [150]
From a SR: 1 additional disc herniation or CES in 3.7 million manipulations (in pts, with lumbar disc herniation).

From their own estimation: $<1$ worsening LDH or CES in 3,72 million manipulations (in pts. with lumbar disc herniation), 1 worsening lumbar disc herniation or CES in 1,78 million manipulations (including manipulations under anesthesia; in pts. with lumbar disc herniation).

From other reviews: 1 CES in 128 million manipulations (given the quality score 84\%), 1 CES in 100 million manipulations (given the quality score $86 \%$ ), $<1$ (CES or herniation) in 1 million manipulations (given the quality score 74\%), 1 LDH or CES in 2,789,709 manipulations (1 LDH in 8,369,129 manipulations, and 1 CES in 4,184,564 manipulations) (given the quality score $32 \%$ ).

From a retrospective study: They stated they were $95 \%$ confident that the risk of complication of manipulation for patients with back pain and sciatica was between $0 \%$ and $5 \%$.'

From a prospective study: 'A prospective evaluation of 2000 patients attending a chiropractic college clinic failed to reveal even one major complication', '1000 new patients and 4700 treatments and found no permanent complications'.

From surveys: 1 minor or transient complication but no serious or permanent complications in 38,137 lumbar spinal manipulations.

From pooling the prospective and retrospective studies together: 0 major, serious, or permanent complications in $>2100$ patients (>13,100 treatments). 0 complications in 117 patients diagnosed as having LDH (>2000 spinal manipulation of probable disc herniations).

From SRs: 1 serious complication in 20,000 to 5 serious complications in 10,000,000 cervical spine manipulations (rated as low accuracy and level $V$ evidence), 1 stroke from cervical manipulation in 100,000 (0.001\%).

From a survey: 1 CVA in 228,050 manipulations, 1 CVA in 1.3 million, 5 CVA in one million.

Their own summarisation: 'Estimates of the incidence of serious complications range from 1 per 2 million manipulations to 1 per 400,000'.

From reviews and a letter: 1 SAE per 1-2 million treatments.

From surveys: 1 sleight neurologic complication per 40,000 manipulations, 1 severe complication per 400,000 manipulations, 1 stroke per 1,300,000 treatments of cervical SMT.

From insurance claim data referred to in a SR: 1 stroke per 2 million manipulations.

From a CC: 1.3 VBA within 1 week of treatment in 100,000 pts $<45$ years receiving chiropractic treatment.

Their own conclusion (partly based on the articles not appearing in their result section): From 1 VBA in 20,000 patients to 1 VBA in 1 million cervical manipulations. <1 CES in 1 million treatments.

From a SR: No complications in 1500 patients treated with manipulation in clinical trials.

From surveys: 1 slight neurological complication in 40,000 cases, 1 important complication in 400,000 manipulative procedures, 1 VBA in 228,050 manipulations, $<5$ strokes in 100.000 patients receiving neck manipulations.

From their own estimation: 5-10 VBA or other complications (spinal cord compression, vertebral fracture, tracheal rupture, diaphragm paralysis, internal carotid hematoma, cardiac arrest) in 10,000,000 manipulations, 3-6 major impairment (paralysis, neurologic deficit, other permanent functional impairment) in 10,000,000 manipulations, $<3$ deaths in 10,000,000 manipulations.

From surveys: 1 serious complication in 400,000 to $>1$ million manipulations, 1 CVA accident in 3.85 million cervical spine manipulations.

They compare the incidence rates with NSAID consumption (0.39-3.2 serious gastrointestinal event in 1000 subjects) and cervical spine surgery (15.6 neurologic complications (spinal cord or nerve root injury, recurrent laryngeal nerve palsy, dural leak, and injury to cervical sympathetic nerve trunk (Horner's syndrome)) in 1000 surgeries and 6.9 deaths in 1000 surgeries. 
Table 5 Estimates for the incidence of serious adverse events following spinal manipulative therapy (Continued)

1995 Dabbs V. \& Lauretti W. J. [114]

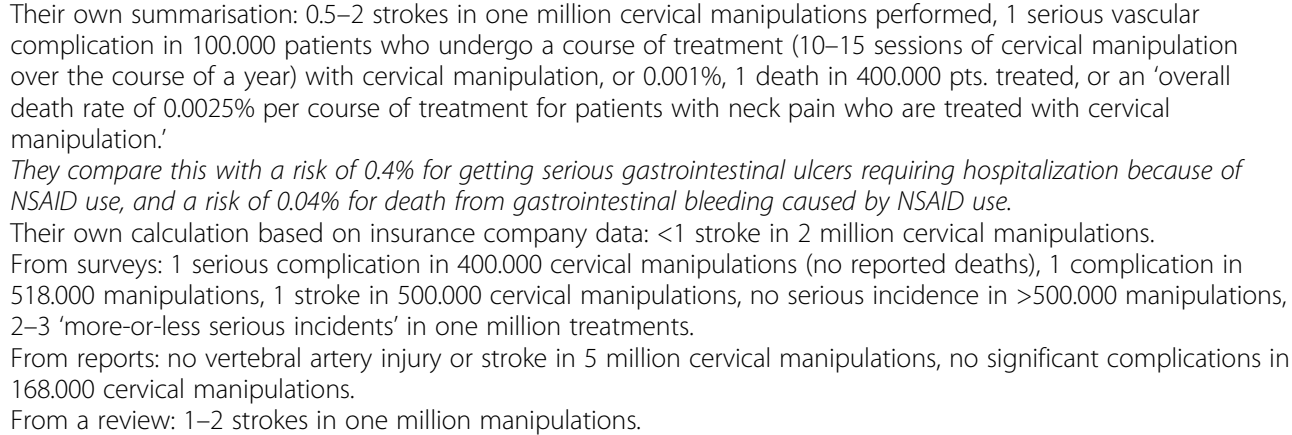

Their own summarisation: 0.5-2 strokes in one million cervical manipulations performed, 1 serious vascular complication in 100.000 patients who undergo a course of treatment (10-15 sessions of cervical manipulation over the course of a year) with cervical manipulation, or $0.001 \%, 1$ death in 400.000 pts. treated, or an 'overall death rate of $0.0025 \%$ per course of treatment for patients with neck pain who are treated with cervical manipulation.'

They compare this with a risk of $0.4 \%$ for getting serious gastrointestinal ulcers requiring hospitalization because of NSAID use, and a risk of $0.04 \%$ for death from gastrointestinal bleeding caused by NSAID use.

Their own calculation based on insurance company data: <1 stroke in 2 million cervical manipulations. From surveys: 1 serious complication in 400.000 cervical manipulations (no reported deaths), 1 complication in 518.000 manipulations, 1 stroke in 500.000 cervical manipulations, no serious incidence in $>500.000$ manipulations, 2-3 'more-or-less serious incidents' in one million treatments.

From reports: no vertebral artery injury or stroke in 5 million cervical manipulations, no significant complications in 168.000 cervical manipulations.

From a review: 1-2 strokes in one million manipulations.

1992 Shekelle P. G. et al.[92] Their own estimation: <1 case of CES in 100 million lumbar spinal manipulations.

CC case-control study, CES cauda equina syndrome, CMT cervical manipulative therapy, CVA cerebrovascular accident, LDH lumbar disc herniation, NSAID nonsteroidal anti-inflammatory drug, $p$ Cohort prospective cohort study, $R C T$ randomized controlled trial, SAE serious adverse event, SMT spinal manipulative therapy, $S R$ systematic review, VAD vertebral artery dissection, VBA vertebrobasilar accident

large extent be unpredictable [155] and have major impact on not only the individual but also the SMT provider and society, it is not possible to ascertain the riskbenefit balance based on the current evidence [156]. We strongly encourage efforts to illuminate the risk-benefit ratio reliably, since this would be of value when comparing SMT with other treatment options. Some of our included reviews indicate that NSAIDs involve a substantially higher risk of SAEs (including death) than SMT $[114,150]$, but they did not take into account the possible benefits.

Table 6 Estimates of the incidences of serious adverse events (some scaled for comparability)

Death
1 in $>3330.000-3,730,000$ manipulations
Stroke
1 in 20,000-2,000,000 manipulations
Vertebrobasilar accident (VBA)
1 in 228,050-1,000,000 manipulations
Cerebrovascular accident (CVA)
1 in 228,050- 3,850,000 manipulations
Lumbar disc herniation (LDH)
1 in 8,369,129 manipulations ${ }^{\text {a }}$
Cauda equina syndrome (CES)
1 CES in $>1,000,000-128,000,000$ manipulations
CES or LDH
1 in $>1,000,000-3,720,000$ manipulations
'Serious adverse events'
1 in 1,000,000-250,000,000 manipulations
'Serious complication'
1 in 20,000-2,000,000 manipulations

${ }^{\mathrm{a}}$ Only one estimate was available
General limitations in overviews are that recently published primary studies or studies not included in reviews cannot be included, the included reviews may overlap, and that the overviews rely on the methodological quality of the included reviews, which again rely on the methodological quality of the primary studies [157]. Considering the low methodological quality of the included reviews, the communicated opinions could possibly be influenced by the background of the authors [158], and by lack of independence between the reviews, i.e. several reviews were written by the same author. A major limitation of this overview was the limited data on AEs and SAEs hindering a synthesis. On the level of reviews, poor reporting of AEs is present [159]; however, even high quality reviews may fail to provide reliable estimates due to poor reporting in the primary studies, and this was frequently highlighted in the discussions of the included reviews. In primary studies, underreporting may be expected for retrospective studies or poorly controlled prospective studies. Including only RCTs would provide an insufficient population size for detecting SAEs reliably, and it has been shown that even in RCTs, AEs and SAEs are poorly reported $[126,160]$ and underreported [96, 161]. Gorrell et al. [162] found that out of 368 RCTs on SMT, only 140 (38\%) reported on AEs. This under-reporting will directly affect the reviews including the studies resulting in a underestimation of the risk. On the other hand, over-reporting may be present, since the different study types (ranging from case reports to RCTs) provide various levels of evidence, and therefore confounding and chance cannot be ruled out as possible explaining factors for some of the observed SAEs associated with SMT.

Our methodological approach has limitations too. Our inclusion criteria were slightly heterogeneous across reviews. We relied on the definitions of SMT 
Table 7 The risk ratio of having the opinion that spinal manipulative therapy is safe or harmful, respectively, if a 'yes' was obtained in the individual AMSTAR items (118 reviews)

\begin{tabular}{|c|c|c|c|c|c|c|}
\hline \multirow[b]{3}{*}{ AMSTAR \#1 } & \multicolumn{6}{|c|}{ Risk ratio (RR) } \\
\hline & \multicolumn{2}{|c|}{ RR (95\% Cl) for communicating that SMT is safe } & \multirow{2}{*}{$\begin{array}{l}P \text { values } \\
0.109\end{array}$} & \multicolumn{2}{|c|}{ RR $(95 \% \mathrm{Cl})$ for communicating that SMT is harmful } & \multirow{2}{*}{$\frac{P \text { values }}{-}$} \\
\hline & 1.4 & (1.0 to 2.1 ) & & Not estimable ${ }^{b}$ & & \\
\hline AMSTAR \#2 & 1.4 & (1.0 to 2.1$)$ & 0.091 & 0.2 & $(0.1$ to 1.0$)$ & 0.025 \\
\hline AMSTAR \#3 & 1.0 & (0.6 to 1.6$)$ & 0.964 & 1.8 & (0.4 to 7.6$)$ & 0.386 \\
\hline AMSTAR \#4 & 1.2 & (0.8 to 1.8$)$ & 0.436 & 0.4 & $(0.1$ to 1.7$)$ & 0.178 \\
\hline AMSTAR \#5 & 1.8 & (1.2 to 2.5$)$ & 0.005 & 0.2 & $(0.0$ to 1.2$)$ & 0.038 \\
\hline AMSTAR \#6 & 1.5 & (0.7 to 3.6$)$ & 0.252 & 1.7 & $(0.2$ to 12.1$)$ & 0.566 \\
\hline AMSTAR \#7 & 3.2 & (1.4 to 7.2 ) & $<0.001$ & 0.1 & (0.0 to 0.2 ) & $<0.001$ \\
\hline AMSTAR \#8 & 1.8 & (1.1 to 3.0 ) & 0.014 & 0.1 & (0.0 to 0.3 ) & $<0.001$ \\
\hline AMSTAR \#9 & 2.2 & (0.8 to 5.8 ) & 0.152 & 0.5 & (0.1 to 3.9 ) & 0.528 \\
\hline AMSTAR \#10 & 2.0 & (1.6 to 2.4 ) & 0.331 & Not estimable $e^{a}$ & & - \\
\hline AMSTAR \#11 & 1.6 & (1.0 to 2.5$)$ & 0.109 & Not estimable $^{b}$ & & - \\
\hline
\end{tabular}

For descriptions of each AMSTAR item, see footnote for Table 4

AMSTAR A Measurement Tool to Assess Systematic Reviews, Cl confidence interval, $R R$ risk ratio, SMT spinal manipulative therapy

${ }^{a}$ No SRs had a 'yes' for this item

${ }^{\mathrm{b}} \mathrm{No}$ SRs had a 'yes' for this item and communicated 'safe'

used by the review authors, which varied between the reviews. Some of the reviews mixed SMT with other interventions under a common category such as 'manual treatment' or 'manipulation' without reporting on only the SMT subgroup. Even when authors describe interventions such as SMT, these may not always include high-velocity, low-amplitude thrusts. In that case, the intervention is less likely to result in SAEs and may influence their and our conclusion about safety by making (high-velocity, low-amplitude thrust-type) SMT appear more safe. Further, we did not require a quality assessment to have been conducted for case reports, case series, cross-sectional studies and surveys, which may have facilitated the inclusion of reviews including only these types of studies. Our judgements regarding the expressed opinions in the reviews were not based on any criteria but based on subjective interpretation and therefore not reproducible even though there was fair agreement between the reviewers. Other limitations include the absence of a double study selection, data extraction and quality assessment, and a very brief protocol. These methodological compromises were taken due to limited time resources. However, our search strategy was broad, and we applied a thorough study selection making us confident that we have identified the vast majority of the relevant scientific literature on SMT and we find it unlikely that more thorough study selection and extraction procedures would result in different conclusions.

\section{Conclusions}

This overview has indeed demonstrated how extensive the literature on SMT is. Unfortunately, the majority of reviews are non-systematic and of poor quality. The available evidence showed a broad range of communicated opinions and very variable estimates of SAE incidence. Reviews with less methodological flaws typically communicated that SMT may be safe; however, the methodological quality was in general low and the included reviews very heterogeneous. Furthermore, for the subset of reviews whose objective was to investigate safety, this could not be replicated. Research of high quality, with sufficient sample size and an appropriate comparison group is needed to obtain reliable risk estimates. Furthermore, reviews suggested that a causal relationship between SMT and SAEs was

Table 8 Assessments of the likelihood of the causal relationship between spinal manipulative therapy and serious adverse events in reviews based on case reports and case series

\begin{tabular}{llllll}
\hline Rating of causal relationship & Ernst 2007 [118] & Ernst 2005 [133] & Ernst 2004 [89] & Ernst 2003 [90] & Stevinson 2002 [124] \\
\hline 'Certain', $n(\%)$ & $8(21.6 \%)$ & $6(42.9 \%)$ & $12(30 \%)$ & $0(0 \%)$ & $5(22.7 \%)$ \\
'Likely', $n(\%)$ & $18(48.6 \%)$ & $6(42.9 \%)$ & $16(40 \%)$ & $0(0 \%)$ & $14(63.6 \%)$ \\
'Possible', $n(\%)$ & $8(21.6 \%)$ & $2(14.3 \%)$ & $9(22.5 \%)$ & $2(100 \%)$ & $0(0 \%)$ \\
'Not assessable' or '???', $n(\%)$ & $3(8.2 \%)$ & $0(0 \%)$ & $3(7.5 \%)$ & $0(0 \%)$ & $3(13.6 \%)$ \\
Total, $n(\%)$ & $37(100 \%)$ & $14(100 \%)$ & $40(100 \%)$ & $2(100 \%)$ & $22(100 \%)$ \\
\hline
\end{tabular}


often not certain. However, the types of SAEs reported were indeed significant, sustaining that there is some risk present; sometimes SMT may even lead to death or permanent disability.

\section{Additional files}

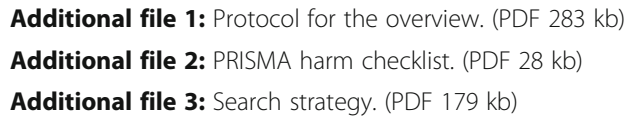

Additional file 6: Table showing further study characteristics including conclusions extracted from each reviews. (PDF $416 \mathrm{~kb}$ )

Additional file 7: Table showing which studies the estimates for the incidence of SAEs are based on. (DOCX $25 \mathrm{~kb}$ )

Additional file 8: Table showing the calculations of RRs of having the opinion that spinal manipulative therapy is safe or harmful, respectively, if a 'yes' was obtained in the individual AMSTAR items, for the 33 reviews, whose objective was to investigate adverse events. (PDF $186 \mathrm{~kb}$ )

\section{Abbreviations}

AEs: Adverse events; AMSTAR: A measurement tool to assess systematic reviews; DARE: Cochrane database of abstracts of reviews of effects; HTA: Cochrane health technology assessment database; PROSPERO: The International Prospective Register of Systematic Reviews; RCTs: Randomized controlled trials; RRs: Risk ratios; SAEs: Serious adverse events; SMT: Spinal manipulative therapy

\section{Acknowledgements}

Not applicable.

\section{Funding}

This work was supported by the Association of Danish Physiotherapists and by The Oak Foundation. The Parker Institute, Bispebjerg and Frederiksberg Hospital is supported by a core grant from the Oak Foundation (OCAY-13-309). The funders had no role in the study design; in the collection, analysis, and interpretation of data; in the writing of the report; or in the decision to submit the article for publication.

\section{Availability of data and materials}

The majority of the data generated and/or analysed during this study are included in this published article and its supplementary information files; the remaining data are available from the corresponding author on reasonable request.

\section{Authors' contributions}

SMN, ST, RC, HB and MH contributed to the design of this overview. SMN performed the study selection, data extraction, and risk of bias assessment, assisted by $\mathrm{MH}$ and LK. SMN, MH and RC analysed and interpreted the data. SMN wrote first draft of the paper. All authors have read and approved the final manuscript.

\section{Competing interests}

$\mathrm{MH}$ is a member of the Association of Danish Physiotherapists that could benefit from this publication; no other relationships or activities that could appear to have influenced the submitted work.

\section{Consent for publication}

Not applicable.

Ethics approval and consent to participate Not applicable.

\section{Publisher's Note}

Springer Nature remains neutral with regard to jurisdictional claims in published maps and institutional affiliations.

\section{Author details}

${ }^{1}$ The Parker Institute, Copenhagen University Hospital, Frederiksberg \& Bispebjerg, Frederiksberg 2000, Denmark. ${ }^{2}$ Clinical Research Unit, The Parker Institute, Copenhagen University Hospital, Frederiksberg \& Bispebjerg, Frederiksberg 2000, Denmark. ${ }^{3}$ Physiotherapy and Biomechanics Research Unit, The Parker Institute, Copenhagen University Hospital, Frederiksberg \& Bispebjerg, Frederiksberg 2000, Denmark.

Received: 21 November 2016 Accepted: 15 March 2017

Published online: 24 March 2017

\section{References}

1. Vernon $\mathrm{H}$, Mrozek J. A revised definition of manipulation. J Manipulative Physiol Ther. 2005;28:68-72.

2. Brodeur R. The audible release associated with joint manipulation. J Manipulative Physiol Ther. 1995;18:155-64.

3. Reggars JW. The manipulative crack. Frequency analysis. Australas Chiropr Osteopathy. 1996:5:39-44.

4. Pettman E. A history of manipulative therapy. J Man Manip Ther. 2007; 15:165-74.

5. Koes BW, van Tulder M, Lin CW, Macedo LG, McAuley J, Maher C. An updated overview of clinical guidelines for the management of non-specific low back pain in primary care. Eur Spine J. 2010;19:2075-94.

6. Gordon R, Cremata E, Hawk C. Guidelines for the practice and performance of manipulation under anesthesia. Chiropr Man Therap. 2014;22:7.

7. Hawk C, Schneider M, Ferrance RJ, Hewitt E, Van Loon M, Tanis L. Best practices recommendations for chiropractic care for infants, children, and adolescents: results of a consensus process. J Manipulative Physiol Ther. 2009;32:639-47.

8. Globe G, Farabaugh RJ, Hawk C, Morris CE, Baker G, Whalen WM, Walters S, Kaeser M, Dehen M, Augat T. Clinical practice guideline: chiropractic care for low back pain. J Manipulative Physiol Ther. 2016;39:1-22.

9. Bryans R, Decina P, Descarreaux M, Duranleau M, Marcoux H, Potter B, Ruegg RP, Shaw L, Watkin R, White E. Evidence-based guidelines for the chiropractic treatment of adults with neck pain. J Manipulative Physiol Ther. 2014;37:42-63.

10. Bryans R, Descarreaux M, Duranleau M, Marcoux H, Potter B, Ruegg R, Shaw L, Watkin R, White E. Evidence-based guidelines for the chiropractic treatment of adults with headache. J Manipulative Physiol Ther. 2011;34:274-89.

11. Hurwitz EL, Chiang LM. A comparative analysis of chiropractic and general practitioner patients in North America: findings from the joint Canada/ United States survey of health, 2002-03. BMC Health Serv Res. 2006;6:49.

12. Hurwitz EL, Coulter ID, Adams AH, Genovese BJ, Shekelle PG. Use of chiropractic services from 1985 through 1991 in the United States and Canada. Am J Public Health. 1998;88:771-6.

13. Hurwitz EL. Epidemiology: spinal manipulation utilization. J Electromyogr Kinesiol. 2012;22:648-54.

14. Rubinstein Sidney M, van Middelkoop M, Assendelft Willem JJ, de Boer Michiel R, van Tulder Maurits W. Spinal manipulative therapy for chronic low-back pain. Cochrane Database Syst Rev. 2011;(2):CD008112. doi:10.1002/ 14651858.CD008112.pub2.

15. Rubinstein SM, Terwee CB, Assendelft WJJ, de Boer MR, van Tulder MW. Spinal manipulative therapy for acute low-back pain. Cochrane Database Syst Rev. 2012;(9):CD008880. doi:10.1002/14651858.CD008880.pub2.

16. Proctor M, Hing W, Johnson Trina C, Murphy Patricia A, Brown J. Spinal manipulation for dysmenorrhoea. Cochrane Database Syst Rev. 2006;(3): CD002119. doi:10.1002/14651858.CD002119.pub3.

17. Hondras Maria A, Linde K, Jones Arthur P. Manual therapy for asthma. Cochrane Database Syst Rev. 2005:(2):CD001002. doi:10.1002/14651858. CD001002.pub2.

18. Cagnie B, Vinck E, Beernaert A, Cambier D. How common are side effects of spinal manipulation and can these side effects be predicted? Man Ther. 2004;9:151-6.

19. Rydell N, Raf L. Spinal manipulation — treatment associated with a high risk of complications. Lakartidningen. 1999;96:3536-40. 
20. Balblanc JC, Pretot C, Ziegler F. Vascular complication involving the conus medullaris or cauda equina after vertebral manipulation for an L4-L5 disk herniation. Rev Rhum Engl Ed. 1998;65:279-82.

21. Peters M, Bohl J, Thomke F, Kallen KJ, Mahlzahn K, Wandel E, Meyer zum Buschenfelde $\mathrm{KH}$. Dissection of the internal carotid artery after chiropractic manipulation of the neck. Neurology. 1995;45:2284-6.

22. Cortazzo JM, Tom KB. Vertebral artery dissection following chiropractic manipulation. Am J Emerg Med. 1998;16:619-20.

23. Carlesso LC, Cairney J, Dolovich L, Hoogenes J. Defining adverse events in manual therapy: an exploratory qualitative analysis of the patient perspective. Man Ther. 2011;16:440-6

24. Cassidy JD, Boyle E, Cote P, He Y, Hogg-Johnson S, Silver FL, Bondy SJ. Risk of vertebrobasilar stroke and chiropractic care: results of a population-based casecontrol and case-crossover study. Spine (Phila Pa 1976). 2008;33:S176-183.

25. Cassidy JD, Boyle E, Cote P, Hogg-Johnson S, Bondy SJ, Haldeman S. Risk of Carotid Stroke after Chiropractic Care: A Population-Based Case-Crossover Study. J Stroke Cerebrovasc Dis. 2016. doi:10.1016/j.jstrokecerebrovasdis.2016.10.031.

26. Smith WS, Johnston SC, Skalabrin EJ, Weaver M, Azari P, Albers GW, Gress DR. Spinal manipulative therapy is an independent risk factor for vertebral artery dissection. Neurology. 2003;60:1424-8.

27. Zorzela L, Loke YK, loannidis JP, Golder S, Santaguida P, Altman DG, Moher D, Vohra S. PRISMA harms checklist: improving harms reporting in systematic reviews. BMJ. 2016;352:1157.

28. Shea BJ, Hamel C, Wells GA, Bouter LM, Kristjansson E, Grimshaw J, Henry DA, Boers M. AMSTAR is a reliable and valid measurement tool to assess the methodological quality of systematic reviews. J Clin Epidemiol. 2009:62:1013-20.

29. Shea BJ, Grimshaw JM, Wells GA, Boers M, Andersson N, Hamel C, Porter AC, Tugwell P, Moher D, Bouter LM. Development of AMSTAR: a measurement tool to assess the methodological quality of systematic reviews. BMC Med Res Methodol. 2007;7:10

30. Mickan S, Tilson JK, Atherton H, Roberts NW, Heneghan C. Evidence of effectiveness of health care professionals using handheld computers: a scoping review of systematic reviews. J Med Internet Res. 2013;15:e212.

31. Bobrovitz N, Onakpoya I, Roberts N, Heneghan C, Mahtani KR. Protocol for an overview of systematic reviews of interventions to reduce unscheduled hospital admissions among adults. BMJ Open. 2015;5:e008269.

32. Edwards IR, Aronson JK. Adverse drug reactions: definitions, diagnosis, and management. Lancet. 2000;356:1255-9.

33. Higgins J, Deeks J. Chapter 7.2.6: measuring agreement. In: Higgins JPT, Deeks JJ, editors. Cochrane Handbook for Systematic Reviews of Interventions Version 510 (updated March 2011). 2011.

34. Xin Z, Xue-Ting L, De-Ying K. GRADE in systematic reviews of acupuncture for stroke rehabilitation: recommendations based on high-quality evidence. Sci Rep. 2015;5:16582.

35. Liddle Sarah D, Pennick V. Interventions for preventing and treating lowback and pelvic pain during pregnancy. Cochrane Database Syst Rev. 2015; (9):CD001139. doi:10.1002/14651858.CD001139.pub4

36. Gross A, Langevin P, Burnie SJ, Bedard-Brochu MS, Empey B, Dugas E, FaberDobrescu M, Andres C, Graham N, Goldsmith CH, et al. Manipulation and mobilisation for neck pain contrasted against an inactive control or another active treatment. Cochrane Database Syst Rev. 2015;(9):CD004249. doi:10. 1002/14651858.CD004249.pub4

37. Page Matthew J, Green S, Kramer S, Johnston Renea V, McBain B, Chau M, Buchbinder R. Manual therapy and exercise for adhesive capsulitis (frozen shoulder). Cochrane Database Syst Rev. 2014;(8):CD011275. doi:10.1002/ 14651858.CD011275

38. Yang M, Yan Y, Yin X, Wang BY, Wu T, Liu GJ, Dong BR. Chest physiotherapy for pneumonia in adults. Cochrane Database Syst Rev. 2013;(2):CD006338. doi:10.1002/14651858.CD006338.pub3.

39. Rubinstein SM, Terwee CB, Assendelft WJ, de Boer MR, van Tulder MW Spinal manipulative therapy for acute low back pain: an update of the cochrane review. Spine (Phila Pa 1976). 2013;38:E158-177.

40. Dobson D, Lucassen Peter LBJ, Miller Joyce J, Vlieger Arine M, Prescott P Lewith G. Manipulative therapies for infantile colic. Cochrane Database Syst Rev. 2012;(12):CD004796. doi:10.1002/14651858.CD004796.pub2.

41. Walker BF, French SD, Grant W, Green S. A Cochrane review of combined chiropractic interventions for low-back pain. Spine (Phila Pa 1976). 2011;36:230-42.

42. Rubinstein SM, van Middelkoop M, Assendelft WJ, de Boer MR, van Tulder MW. Spinal manipulative therapy for chronic low-back pain: an update of a Cochrane review. Spine (Phila Pa 1976). 2011;36:E825-846.
43. Huang T, Shu X, Huang YS, Cheuk DK. Complementary and miscellaneous interventions for nocturnal enuresis in children. Cochrane Database Syst Rev. 2011;(12):CD005230. doi:10.1002/14651858.CD005230.pub2.

44. Walker BF, French SD, Grant W, Green S. Combined chiropractic interventions for low-back pain. Cochrane Database Syst Rev. 2010;(4): CD005427. doi:10.1002/14651858.CD005427.pub2.

45. Brønfort G, Nilsson N, Haas M, Evans R, Goldsmith CH, Assendelft WJ, Bouter LM. Non-invasive physical treatments for chronic/recurrent headache. Cochrane Database Syst Rev. 2004;(3):CD001878. doi:10.1002/14651858. CD001878.pub2.

46. Page Matthew J, Green S, McBain B, Surace Stephen J, Deitch J, Lyttle N, Mrocki Marshall A, Buchbinder R. Manual therapy and exercise for rotator cuff disease. Cochrane Database Syst Rev. 2016;(6):CD012224. doi:10.1002/ 14651858.CD012224.

47. Zhu L, Wei $X$, Wang S. Does cervical spine manipulation reduce pain in people with degenerative cervical radiculopathy? A systematic review of the evidence, and a meta-analysis. Clin Rehabil. 2016:30(2):145-55.

48. Yuan QL, Guo TM, Liu L, Sun F, Zhang YG. Traditional Chinese medicine for neck pain and low back pain: a systematic review and meta-analysis. PLoS One. 2015;10, e0117146

49. Southerst D, Marchand AA, Cote P, Shearer HM, Wong JJ, Varatharajan S, Randhawa K, Sutton D, Yu H, Gross DP, et al. The effectiveness of noninvasive interventions for musculoskeletal thoracic spine and chest wall pain: a systematic review by the Ontario Protocol for Traffic Injury Management (OPTIMa) Collaboration. J Manipulative Physiol Ther. 2015;38:521-31.

50. Sutton DA, Cote P, Wong JJ, Varatharajan S, Randhawa KA, Yu H, Southerst D, Shearer HM, van der Velde GM, Nordin MC, et al. Is multimodal care effective for the management of patients with whiplash-associated disorders or neck pain and associated disorders? A systematic review by the Ontario Protocol for Traffic Injury Management (OPTIMa) Collaboration. Spine J. 2016;16(12):1541-65

51. Kizhakkeveettil A, Rose K, Kadar GE. Integrative therapies for low back pain that include complementary and alternative medicine care: a systematic review. Glob Adv Health Med. 2014:3:49-64.

52. Franke H, Franke JD, Fryer G. Osteopathic manipulative treatment for nonspecific low back pain: a systematic review and meta-analysis. BMC Musculoskelet Disord. 2014;15:286.

53. Close C, Sinclair M, Liddle SD, Madden E, McCullough JE, Hughes C. A systematic review investigating the effectiveness of Complementary and Alternative Medicine (CAM) for the management of low back and/or pelvic pain (LBPP) in pregnancy. J Adv Nurs. 2014;70:1702-16.

54. Schroeder J, Kaplan L, Fischer DJ, Skelly AC. The outcomes of manipulation or mobilization therapy compared with physical therapy or exercise for neck pain: a systematic review. Evid Based Spine Care J. 2013;4:30-41.

55. Scholten-Peeters GG, Thoomes E, Konings S, Beijer M, Verkerk K, Koes BW Verhagen AP. Is manipulative therapy more effective than sham manipulation in adults: a systematic review and meta-analysis. Chiropr Man Therap. 2013;21:34

56. Posadzki $\mathrm{P}$, Lee MS, Ernst E. Osteopathic manipulative treatment for pediatric conditions: a systematic review. Pediatrics. 2013;132:140-52.

57. Huisman PA, Speksnijder CM, de Wijer A. The effect of thoracic spine manipulation on pain and disability in patients with non-specific neck pain: a systematic review. Disabil Rehabil. 2013:35:1677-85.

58. Posadzki P, Ernst E. Spinal manipulations for tension-type headaches: a systematic review of randomized controlled trials. Complement Ther Med. 2012;20:232-9

59. Lin JH, Chiu TT, Hu J. Chinese manipulation for mechanical neck pain: a systematic review. Clin Rehabil. 2012;26:963-73.

60. Kuczynski JJ, Schwieterman B, Columber K, Knupp D, Shaub L, Cook CE. Effectiveness of physical therapist administered spinal manipulation for the treatment of low back pain: a systematic review of the literature. Int J Sports Phys Ther. 2012;7:647-62.

61. Posadzki P, Ernst E. Systematic reviews of spinal manipulations for headaches: an attempt to clear up the confusion. Headache. 2011:51:1419-25.

62. Posadzki P, Ernst E. Spinal manipulations for the treatment of migraine: a systematic review of randomized clinical trials. Cephalalgia. 2011;31:964-70.

63. Posadzki P, Ernst E. Spinal manipulations for cervicogenic headaches: a systematic review of randomized clinical trials. Headache. 2011;51:1132-9.

64. Posadzki P, Ernst E. Osteopathy for musculoskeletal pain patients: a systematic review of randomized controlled trials. Clin Rheumatol. 2011;30:285-91. 
65. Cross KM, Kuenze C, Grindstaff TL, Hertel J. Thoracic spine thrust manipulation improves pain, range of motion, and self-reported function in patients with mechanical neck pain: a systematic review. J Orthop Sports Phys Ther. 2011;41:633-42

66. Hahne AJ, Ford JJ, McMeeken JM. Conservative management of lumbar disc herniation with associated radiculopathy: a systematic review. Spine (Phila Pa 1976). 2010;35:E488-504

67. Hunt KJ, Hung SK, Boddy K, Ernst E. Chiropractic manipulation for carpal tunnel syndrome: a systematic review. Hand Therapy. 2009;14:89-94.

68. Vernon $\mathrm{H}$, Humphreys BK. Chronic mechanical neck pain in adults treated by manual therapy: a systematic review of change scores in randomized controlled trials of a single session. J Man Manip Ther. 2008;16:E42-52.

69. Vernon $H$, Humphreys BK. Manual therapy for neck pain: an overview of randomized clinical trials and systematic reviews. Eura Medicophys. 2007:43:91-118.

70. Luijsterburg PA, Verhagen AP, Ostelo RW, Os TA, Peul WC, Koes BW. Effectiveness of conservative treatments for the lumbosacral radicular syndrome: a systematic review. Eur Spine J. 2007;16:881-99.

71. Gross AR, Goldsmith C, Hoving JL, Haines T, Peloso P, Aker P, Santaguida P, Myers $C$. Conservative management of mechanical neck disorders: a systematic review. J Rheumatol. 2007;34:1083-102.

72. Lenssinck ML, Damen L, Verhagen AP, Berger MY, Passchier J, Koes BW. The effectiveness of physiotherapy and manipulation in patients with tensiontype headache: a systematic review. Pain. 2004;112:381-8.

73. Gross AR, Kay T, Hondras M, Goldsmith C, Haines T, Peloso P, Kennedy C, Hoving J. Manual therapy for mechanical neck disorders: a systematic review. Man Ther. 2002;7:131-49.

74. Gerritsen AA, Krom MC, Struijs MA, Scholten RJ, Vet HC, Bouter LM. Conservative treatment options for carpal tunnel syndrome: a systematic review of randomised controlled trials. J Neurol. 2002;249:272-80.

75. Ernst E, Harkness E. Spinal manipulation: a systematic review of shamcontrolled, double-blind, randomized clinical trials. J Pain Symptom Manage. 2001;22:879-89.

76. Bronfort G, Assendelft WJ, Evans R, Haas M, Bouter L. Efficacy of spinal manipulation for chronic headache: a systematic review. J Manipulative Physiol Ther. 2001;24:457-66.

77. Ernst E. Does spinal manipulation have specific treatment effects? Fam Pract. 2000;17:554-6.

78. Vernon H, McDermaid CS, Hagino C. Systematic review of randomized clinical trials of complementary/alternative therapies in the treatment of tension-type and cervicogenic headache. Complement Ther Med. 1999;7:142-55.

79. Aker PD, Gross AR, Goldsmith CH, Peloso P. Conservative management of mechanical neck pain: systematic overview and meta-analysis. BMJ. 1996; 313:1291-6.

80. Blanchette MA, Stochkendahl MJ, Borges Da Silva R, Boruff J, Harrison P, Bussieres A. Effectiveness and economic evaluation of chiropractic care for the treatment of low back pain: a systematic review of pragmatic studies. PLoS One. 2016;11, e0160037.

81. Cerritelli F, Ruffini N, Lacorte E, Vanacore N. Osteopathic manipulative treatment in neurological diseases: systematic review of the literature. J Neurol Sci. 2016:369:333-41.

82. Franke H, Franke JD, Fryer G. Osteopathic manipulative treatment for chronic nonspecific neck pain: a systematic review and meta-analysis. Int J Osteopath Med. 2015;18:255-67.

83. Hall H, Cramer H, Sundberg T, Ward L, Adams J, Moore C, Sibbritt D, Lauche $R$. The effectiveness of complementary manual therapies for pregnancyrelated back and pelvic pain: a systematic review with meta-analysis. Medicine (Baltimore). 2016;95, e4723.

84. Ruddock JK, Sallis H, Ness A, Perry RE. Spinal manipulation vs sham manipulation for nonspecific low back pain: a systematic review and metaanalysis. J Chiropr Med. 2016;15:165-83.

85. Varatharajan S, Ferguson B, Chrobak K, Shergill Y, Cote P, Wong JJ, Yu H, Shearer HM, Southerst D, Sutton D, et al. Are non-invasive interventions effective for the management of headaches associated with neck pain? An update of the Bone and Joint Decade Task Force on Neck Pain and Its Associated Disorders by the Ontario Protocol for Traffic Injury Management (OPTIMa) Collaboration. Eur Spine J. 2016;25:1971-99.

86. Wong JJ, Shearer HM, Mior S, Jacobs C, Cote P, Randhawa K, Yu H, Southerst D, Varatharajan S, Sutton D, et al. Are manual therapies, passive physical modalities, or acupuncture effective for the management of patients with whiplash-associated disorders or neck pain and associated disorders? An update of the Bone and Joint Decade Task Force on Neck Pain and Its Associated Disorders by the OPTIMa collaboration. Spine J. 2016;16:1598-630

87. Yao M, Sun YL, Dun RL, Lan TY, Li JL, Lee HJ, Haraguchi N, Wang YJ, Cui XJ. Is manipulative therapy clinically necessary for relief of neck pain? A systematic review and meta-analysis. Chin J Integr Med. 2016. doi:10.1007/ s1 1655-016-2506-1.

88. Carnes D, Mars TS, Mullinger B, Froud R, Underwood M. Adverse events and manual therapy: a systematic review. Man Ther. 2010;15:355-63.

89. Ernst E. Cerebrovascular complications associated with spinal manipulation. Phys Ther Rev. 2004;9:5-15.

90. Ernst E. Serious adverse effects of unconventional therapies for children and adolescents: a systematic review of recent evidence. Eur J Pediatr. 2003; 162:72-80

91. Rubinstein SM, Peerdeman SM, van Tulder MW, Riphagen I, Haldeman S. A systematic review of the risk factors for cervical artery dissection. Stroke. 2005;36:1575-80.

92. Shekelle PG, Adams AH, Chassin MR, Hurwitz EL, Brook RH. Spinal manipulation for low-back pain. Ann Intern Med. 1992;117:590-8.

93. Yin P, Gao N, Wu J, Litscher G, Xu S. Adverse events of massage therapy in pain-related conditions: a systematic review. Evid Based Complement Alternat Med. 2014;2014:480956.

94. Khorsan R, Hawk C, Lisi AJ, Kizhakkeveettil A. Manipulative therapy for pregnancy and related conditions: a systematic review. Obstet Gynecol Surv. 2009;64:416-27.

95. Shin BC, Lee MS, Park TY, Ernst E. Serious adverse events after spinal manipulation: a systematic review of the Korean literature. Focus Altern Complement Ther. 2010;15:198-201.

96. Carlesso LC, Gross AR, Santaguida PL, Burnie S, Voth S, Sadi J. Adverse events associated with the use of cervical manipulation and mobilization for the treatment of neck pain in adults: a systematic review. Man Ther. 2010;15:434-44.

97. Brantingham JW, Cassa TK, Bonnefin D, Pribicevic M, Robb A, Pollard H, Tong V, Korporaal C. Manipulative and multimodal therapy for upper extremity and temporomandibular disorders: a systematic review. J Manipulative Physiol Ther. 2013;36:143-201.

98. Todd AJ, Carroll MT, Robinson A, Mitchell EK. Adverse Events Due to Chiropractic and Other Manual Therapies for Infants and Children: A Review of the Literature. J Manipulative Physiol Ther. 2015;38(9):699-712.

99. Gouveia LO, Castanho P, Ferreira JJ. Safety of chiropractic interventions: a systematic review. Spine (Phila Pa 1976). 2009;34:E405-413.

100. Hawk C, Khorsan R, Lisi AJ, Ferrance RJ, Evans MW. Chiropractic care for nonmusculoskeletal conditions: a systematic review with implications for whole systems research. J Altern Complement Med. 2007:13:491-512.

101. Clar C, Tsertsvadze A, Court R, Hundt GL, Clarke A, Sutcliffe P. Clinical effectiveness of manual therapy for the management of musculoskeletal and non-musculoskeletal conditions: systematic review and update of UK evidence report. Chiropr Man Therap. 2014;22:12.

102. Brantingham JW, Bonnefin D, Perle SM, Cassa TK, Globe G, Pribicevic M, Hicks M, Korporaal C. Manipulative therapy for lower extremity conditions: update of a literature review. J Manipulative Physiol Ther. 2012:35:127-66

103. Hebert JJ, Stomski NJ, French SD, Rubinstein SM. Serious Adverse Events and Spinal Manipulative Therapy of the Low Back Region: A Systematic Review of Cases. J Manipulative Physiol Ther. 2015;38(9):677-91.

104. Brantingham JW, Cassa TK, Bonnefin D, Jensen M, Globe G, Hicks M, Korporaal C. Manipulative therapy for shoulder pain and disorders: expansion of a systematic review. J Manipulative Physiol Ther. 2011;34:314-46.

105. Young JL, Walker D, Snyder S, Daly K. Thoracic manipulation versus mobilization in patients with mechanical neck pain: a systematic review. J Man Manip Ther. 2014:22:141-53.

106. Gleberzon BJ, Arts J, Mei A, McManus EL. The use of spinal manipulative therapy for pediatric health conditions: a systematic review of the literature. J Can Chiropr Assoc. 2012;56:128-41.

107. Stuber KJ, Smith DL. Chiropractic treatment of pregnancy-related low back pain: a systematic review of the evidence. J Manipulative Physiol Ther. 2008; 31:447-54.

108. Kaminskyj A, Frazier M, Johnstone K, Gleberzon BJ. Chiropractic care for patients with asthma: a systematic review of the literature. J Can Chiropr Assoc. 2010;54:24-32. 
109. Parkinson L, Sibbritt D, Bolton P, van Rotterdam J, Villadsen I. Well-being outcomes of chiropractic intervention for lower back pain: a systematic review. Clin Rheumatol. 2013;32:167-80.

110. Reiman MP, Harris JY, Cleland JA. Manual therapy interventions for patients with lumbar spinal stenosis: a systematic review. N Z J Physiother. 2009;37:17-28.

111. Gemmell H, Miller P. Comparative effectiveness of manipulation, mobilisation and the activator instrument in treatment of non-specific neck pain: a systematic review. Chiropr Osteopat. 2006;14:7.

112. Lystad RP, Bell G, Bonnevie-Svendsen M, Carter CV. Manual therapy with and without vestibular rehabilitation for cervicogenic dizziness: a systematic review. Chiropr Man Therap. 2011;19:21.

113. Vernon H, Humphreys K, Hagino C. Chronic mechanical neck pain in adults treated by manual therapy: a systematic review of change scores in randomized clinical trials. J Manipulative Physiol Ther. 2007;30:215-27.

114. Dabbs V, Lauretti WJ. A risk assessment of cervical manipulation vs. NSAIDs for the treatment of neck pain. J Manipulative Physiol Ther. 1995;18:530-6.

115. Oliphant D. Safety of spinal manipulation in the treatment of lumbar disk herniations: a systematic review and risk assessment. J Manipulative Physiol Ther. 2004;27:197-210.

116. Cicchitti L, Martelli M, Cerritelli F. Chronic inflammatory disease and osteopathy: a systematic review. PLoS One. 2015:10, e0121327.

117. Magee DJ, Oborn-Barrett E, Turner S, Fenning N. A systematic overview of the effectiveness of physical therapy intervention on soft tissue neck injury following trauma. Physiotherapy Canada. 2000;52:111-30.

118. Ernst E. Adverse effects of spinal manipulation: a systematic review. J R Soc Med. 2007;100:330-8.

119. Ernst E. Adverse effects of unconventional therapies in the elderly: a systematic review of the recent literature. J Am Aging Assoc. 2002;25:11-20.

120. Lisi AJ, Holmes EJ, Ammendolia C. High-velocity low-amplitude spinal manipulation for symptomatic lumbar disk disease: a systematic review of the literature. J Manipulative Physiol Ther. 2005;28:429-42.

121. Fabio RP. Manipulation of the cervical spine: risks and benefits. Phys Ther. 1999;79:50-65

122. Haldeman S, Kohlbeck FJ, McGregor M. Risk factors and precipitating neck movements causing vertebrobasilar artery dissection after cervical trauma and spinal manipulation. Spine (Phila Pa 1976). 1999:24:785-94.

123. Puentedura EJ, March J, Anders J, Perez A, Landers MR, Wallmann HW, Cleland JA. Safety of cervical spine manipulation: are adverse events preventable and are manipulations being performed appropriately? A review of 134 case reports. J Man Manip Ther. 2012;20:66-74.

124. Stevinson C, Ernst E. Risks associated with spinal manipulation. Am J Med. 2002;112:566-71

125. Haynes MJ, Vincent K, Fischhoff C, Bremner AP, Lanlo O, Hankey GJ. Assessing the risk of stroke from neck manipulation: a systematic review. Int J Clin Pract. 2012;66:940-7.

126. Wynd S, Westaway M, Vohra S, Kawchuk G. The quality of reports on cervical arterial dissection following cervical spinal manipulation. PLoS One. 2013;8:e59170.

127. Miley ML, Wellik KE, Wingerchuk DM, Demaerschalk BM. Does cervical manipulative therapy cause vertebral artery dissection and stroke? Neurologist. 2008;14:66-73

128. Ernst E. Deaths after chiropractic: a review of published cases. Int J Clin Pract. 2010:64:1162-5.

129. Ernst E. Prospective investigations into the safety of spinal manipulation. J Pain Symptom Manage. 2001;21:238-42.

130. Tuchin P. A systematic literature review of intracranial hypotension following chiropractic. Int J Clin Pract. 2014;68:396-402.

131. Assendelft WJ, Bouter LM, Knipschild PG. Complications of spinal manipulation: a comprehensive review of the literature. J Fam Pract. 1996;42:475-80.

132. Stuber KJ, Wynd S, Weis CA. Adverse events from spinal manipulation in the pregnant and postpartum periods: a critical review of the literature. Chiropr Man Therap. 2012;20:8

133. Ernst E. Ophthalmological adverse effects of (chiropractic) upper spinal manipulation: evidence from recent case reports. Acta Ophthalmol Scand. 2005;83:581-5

134. Puentedura EJ, O'Grady WH. Safety of thrust joint manipulation in the thoracic spine: a systematic review. J Man Manip Ther. 2015;23:154-61.

135. Snelling NJ. Spinal manipulation in patients with disc herniation: a critical review of risk and benefit. Int J Osteopath Med. 2006;9:77-84.

136. Ernst E. Manipulation of the cervical spine: a systematic review of case reports of serious adverse events, 1995-2001. Med J Aust. 2002;176:376-80.
137. Church EW, Sieg EP, Zalatimo O, Hussain NS, Glantz M, Harbaugh RE. Systematic review and meta-analysis of chiropractic care and cervical artery dissection: no evidence for causation. Cureus. 2016;8, e498.

138. Posadzki P, Albedah AMN, Khalil MMK, Alqaed MS, Lee MS, Ernst E, Car J. Complementary and alternative medicine for the prevention and treatment of migraine headache: an overview of systematic reviews. Focus Altern Complement Ther. 2015;20:58-73.

139. Ruffini N, D'Alessandro G, Cardinali L, Frondaroli F, Cerritelli F. Osteopathic manipulative treatment in gynecology and obstetrics: a systematic review. Complement Ther Med. 2016;26:72-8.

140. Wearing J, Beaumont S, Forbes D, Brown B, Engel R. The use of spinal manipulative therapy in the management of chronic obstructive pulmonary disease: a systematic review. J Altern Complement Med. 2016;22:108-14.

141. Chou R, Huffman L. Evaluation and management of low back pain. Glenview, Illinois: American Pain Society; 2007.

142. Gross AR, Kay TM, Kennedy C, Gasner D, Hurley L, Yardley K, Hendry L, McLaughlin L. Clinical practice guideline on the use of manipulation or mobilization in the treatment of adults with mechanical neck disorders. Man Ther. 2002;7:193-205.

143. Chou R, Huffman LH. Nonpharmacologic therapies for acute and chronic low back pain: a review of the evidence for an American Pain Society/ American College of Physicians clinical practice guideline. Ann Intern Med. 2007;147:492-504.

144. Coulter ID, Hurwitz E, Adams AH, Meeker W, Hansen DT, Mootz R, Aker P, Genovese B, Shekelle PG. The appropriateness of manipulation and mobilization of the cervical spine. Santa Monica: RAND Corporation; 1996.

145. Brurberg KG, Myrhaug HT, Reinar LM. Diagnostics and treatment of infant suspected with kinematic imbalance due to suboccipital strain (KISS). In: Rapport fra Kunnskapssenteret nr 17 - 2009. Oslo: The Norwegian Knowledge Centre for the Health Services (NOKC); 2009.

146. Brown A, Angus D, Chen S, Tang Z, Milne S, Pfaff J, Li H, Mensinkai S. Costs and outcomes of chiropractic treatment for low back pain. In: Health Technology Assessment Database. 2005. p. 88.

147. Boudreau R, Argaez C. Chiropractic interventions for acute or chronic lower back pain in adults: a review of the clinical and cost-effectiveness. In: Health Technology Assessment Database: Canadian Agency for Drugs and Technologies in Health (CADTH). 2009.

148. Furlan AD, Yazdi F, Tsertsvadze A, Gross A, Van Tulder M, Santaguida L, Gagnier J, Ammendolia C, Dryden T, Doucette S, et al. A systematic review and meta-analysis of efficacy, cost-effectiveness, and safety of selected complementary and alternative medicine for neck and low-back pain. Evid Based Complement Alternat Med. 2012;2012:953139.

149. Furlan AD, Yazdi F, Tsertsvadze A, Gross A, Van Tulder M, Santaguida L, Cherkin D, Gagnier J, Ammendolia C, Ansari MT, et al. Complementary and alternative therapies for back pain II. Evidence Report/Technology Assessment No. 194. (Prepared by the University of Ottawa Evidence-based Practice Center under Contract No. 290-2007-10059-I (EPCIII). AHRO Publication No. 10(11) E007. Rockville: Agency for Healthcare Research and Quality; 2010. p. 1-764.

150. Hurwitz EL, Aker PD, Adams AH, Meeker WC, Shekelle PG. Manipulation and mobilization of the cervical spine. A systematic review of the literature. Spine (Phila Pa 1976). 1996;21:1746-59. discussion 1759-1760.

151. Boudreau R, Spry C. Treatment of hydromyelia in adults: a review of clinical effectiveness and guidelines. In: Health Technology Assessment Database. Ottawa: Canadian Agency for Drugs and Technologies in Health (CADTH); 2009.

152. Oduneye F. Spinal manipulation for chronic neck pain. In: Health Technology Assessment Database. 2004. p. 10.

153. Chou R, Deyo R, Friedly J, Skelly A, Hashimoto R, Weimer M, Fu R, Dana T, Kraegel P, Griffin J, et al. Noninvasive treatments for low back pain (Structured abstract). In: Health Technology Assessment Database. Rockville: Agency for Healthcare Research and Quality (AHRQ); 2016.

154. Chou R, Deyo R, Friedly J, Skelly A, Hashimoto R, Weimer M, Fu R, Dana T, Kraegel P, Griffin J, et al. AHRQ comparative effectiveness reviews. In: Noninvasive Treatments for Low Back Pain. Rockville (MD): Agency for Healthcare Research and Quality (US); 2016.

155. Haldeman S, Kohlbeck FJ, McGregor M. Unpredictability of cerebrovascular ischemia associated with cervical spine manipulation therapy: a review of sixty-four cases after cervical spine manipulation. Spine (Phila Pa 1976). 2002;27:49-55.

156. Ernst E. Chiropractic care: attempting a risk-benefit analysis. Am J Public Health. 2002;92:1603-4. 
157. Hartling L, Vandermeer B, Fernandes RM. Systematic reviews, overviews of reviews and comparative effectiveness reviews: a discussion of approaches to knowledge synthesis. Evid Based Child Health. 2014;9:486-94.

158. Haldeman S, Carey P, Townsend M, Papadopoulos C. Clinical perceptions of the risk of vertebral artery dissection after cervical manipulation: the effect of referral bias. Spine J. 2003;2:334-42

159. Zorzela L, Golder S, Liu Y, Pilkington K, Hartling L, Joffe A, Loke Y, Vohra S. Quality of reporting in systematic reviews of adverse events: systematic review. BMJ. 2014;348:f7668.

160. Ernst E, Posadzki P. Reporting of adverse effects in randomised clinical trials of chiropractic manipulations: a systematic review. N Z Med J. 2012;125:87-140.

161. Tang E, Ravaud P, Riveros C, Perrodeau E, Dechartres A. Comparison of serious adverse events posted at ClinicalTrials.gov and published in corresponding journal articles. BMC Med. 2015;13:189.

162. Gorrell LM, Engel RM, Brown B, Lystad RP. The reporting of adverse events following spinal manipulation in randomized clinical trials-a systematic review. Spine J. 2016:16:1143-51.

Submit your next manuscript to BioMed Central and we will help you at every step:

- We accept pre-submission inquiries

- Our selector tool helps you to find the most relevant journal

- We provide round the clock customer support

- Convenient online submission

- Thorough peer review

- Inclusion in PubMed and all major indexing services

- Maximum visibility for your research

Submit your manuscript at www.biomedcentral.com/submit
Biomed Central 